\title{
Intraday Relationship Between Market Activity And Public Announcements
}

Alexis Cellier, University of Paris-Est - IRG, France

Waël Louhichi, University of Rennes I- IAE/IGR-CREM, UMR 6211 CNRS, France

\begin{abstract}
This paper aims to study the relationship between public information arrival and Euronext Paris intraday activity. The information flow is measured as the number of news items recorded by Reuters and conditional volatility is modeled by an EGARCH process. Our results reveal a strong positive relationship between public information flow and trading volume and a moderate positive relation between stock returns volatility and the information flow. These results are available for the CAC4O Index as well as for individual stocks and are robust even after controlling for the impact of the intraday patterns.
\end{abstract}

Keywords: Information flow; conditional volatility; trading volume; market microstructure; intraday analysis; EGARCH

\section{INTRODUCTION}

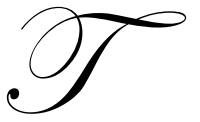

he link between information and price changes is a central issue in the market microstructure literature. Indeed, one of the main objectives of microstructure models is to examine the relationship between information and market activity. The mixture of distribution hypothesis (MDH), has attempted to provide a theoretical justification for this relation. The seminal work of Clark (1973) has introduced the MDH hypothesis, which supposes that stock price changes are driven by information. This hypothesis was extended in the models of Epps and Epps (1976) and Tauchen and Pitts (1983), which highlight a strong relationship between the information flow and market activity. These models consider the information flow as a latent common factor that affects both of trading volume and stock prices. Thus, price changes and trading volume may be correlated as they depend jointly on the intensity of the information flow ( $\mathrm{Li}$ and $\mathrm{Wu}, 2006$ ). Empirically, this means that trading volume and stock prices react contemporaneously in response to information releases. In fact, the arrival of new information to the market induces a price adjustment process through the sequence of trades. This hypothesis is also confirmed by event studies and market efficiency literature ${ }^{1}$.

The aim of our study is to examine the relationship between intraday market activity and the rate of public information flow. However, the literature highlights the difficulty to measure the information flow. Lamoureux and Lastrapes (1990) and Maillet and Michel (1997) propose to use the trading volume as a proxy for information arrival. In this study, we prefer the measure proposed by Berry and Howe (1994). They quantify the information flow as the number of news items published by Reuters per unit of time. The authors break down the trading day into 13 half-hourly intervals and regress the absolute returns against the number of news items published by Reuters during each period. The authors show that the coefficient of the variable "number of news items" is not significant for the 13 regressions. Berry and Howe (1994) conclude to an insignificant relationship between price volatility and the intraday information flow. However, as mentioned by Kalev, Liu, Pharm and Jarnecic (2004), the use of realised volatility measures such as absolute returns can generate biased results regarding the well-known conditional heteroskedasticity of volatility time series. Kalev et al. (2004) focus on the relationship between the information flow and conditional volatility in the Australian market. Using a GARCH model, the authors highlight a strong positive relationship between public information arrival and stock price volatility.

\footnotetext{
${ }^{1}$ For more details see Fama (1991).
} 
In this paper, we propose to shed light on the intraday relationship between the information flow and market activity in Euronext Paris. To measure price movements, we consider the conditional volatility instead of the realized volatility. Unlike, Kalev et al. (2004) we propose to distinguish between overnight announcements and news releases published during the continuous market. This treatment allows us to take into account the specific rules of Euronext Paris at the opening period and at the closing period. Our results reveal a strong positive relationship between the trading volume and the number of news items published by Reuters. But, we find a moderate positive relation between the conditional volatility and the information flow. The remainder of the paper is organised as follows. Section 2 presents the organisation of Euronext Paris, specifies the research methodology and gives a description of the data. Section 3 exposes our empirical results. The last section concludes.

\section{ORGANISATION OF EURONEXT PARIS AND METHODOLOGY}

\subsection{Euronext Paris microstructure}

Euronext was created in September 2000 from the merger of the Amsterdam, Brussels and Paris stock exchanges ${ }^{2}$. Recently, the Euronext group merged with the New York Stock Exchange (NYSE). Euronext Paris operates through a single electronic trading system (Nouveau Système de Cotation: NSC). Traders' orders are conveyed to a central order book. A transaction takes place when a new order is placed and if a matching order exists on the other side of the book. The trading day begins at 7:15 a.m. with a preopening period where traders can place, modify or cancel orders. The market opens at 9:00 a.m. with a call auction, which determines the opening price. From 9:00 a.m. to 5:25 p.m. the market is in its continuous period. In the same way as the opening, the market closes with a preclosing period from 5:25 p.m. to 5:30 p.m. The closing price is determined at 5:30 p.m. with a call auction. A "trading at last" period was introduced to give investors the opportunity to trade after 5:30 p.m. at the closing price. The CAC40 index is the main benchmark for Euronext Paris. It is comprised of the 40 largest and most liquid stocks trading on the exchange.

\subsection{Conditional stock price volatility}

\subsubsection{The EGARCH model}

The clustering pattern of volatility is a well-known phenomenon in the financial literature. In fact, several empirical studies show that volatility time series are characterized by the presence of conditional heteroskedasticity. The family of $\mathrm{ARCH}^{3}$ models accounts for the volatility persistence effect and tries to capture conditional heteroskedasticity patterns. In these models, the current idiosyncratic variance depends on its past levels and past innovations. In this study, we propose using the EGARCH (exponential general auto regressive conditional heteroskedasticity) model proposed by Nelson (1991). The EARCH $(1,1)$ can be presented as follows:

$r_{t}=\mu+\varepsilon_{t}$

$\log \left(\operatorname{Var}\left[\varepsilon_{t} \mid \varepsilon_{t-1}\right]\right)=\log \left(\delta_{t}^{2}\right)=\log \left(h_{t}\right)$

And

$Z_{t-1}=\frac{\varepsilon_{t-1}}{\delta_{t-1}}$

$\log \left(h_{t}\right)=\omega+\gamma Z_{t-1}+\alpha\left(\left|Z_{t-1}\right|-\sqrt{\frac{2}{\pi}}\right)+\beta \log \left(h_{t-1}\right)$

\footnotetext{
${ }^{2}$ Lisbon, Porto and the LIFFE joined the group later.

${ }^{3}$ For more details about these models, see Engle (1982) and Bollerslev (1986). 
Where $r_{t}$ is the level of stock returns at the interval $\mathrm{t}$ and $\mu$ is a constant. The coefficient $\gamma$ accounts for the asymmetry effects. The errors (innovations) $\varepsilon_{t}$ are assumed to be identically and independently distributed. In order to account for this constraint, we compute the variance-covariance matrix using the algorithm of Bollerslev and Wooldridge $^{4}$ (1992). In this case, our estimate will be robust even if returns are not normally distributed. Expression (2) is the equation of the conditional volatility $h_{t}$. The model supposes that the volatility of the current period depends upon the conditional volatility of the former period $h_{t-1}$ and innovation $\varepsilon_{t-1}$.

\subsubsection{The research model}

To test the impact of public information arrival on price volatility, we introduce an informational proxy ( $N_{t}$ ) in the conditional variance equation. This variable represents the number of news items published by Reuters during each 15-minute period t. If the coefficient $\lambda$ of the variable $N_{t}$ is significant, we can conclude that public information arrival has an impact on price volatility. In order to take into account the specific rules of Euronext Paris during the opening period and the closing period, we propose to distinguish between overnight announcements and the news published during the continuous market. First, we test our model during the continuous market. Next, we focus on the impact of overnight information flow on the open-to-close volatility.

The intraday U-shaped pattern of volume and volatility is a well-documented phenomenon in the microstructure literature. Jain and Joh (1985), Wood, McInish and Ord (1985) and Blau, Van Ness and Van Ness (2009) highlight heavy market activity in the beginning and the end of the trading day. To account for this phenomenon, we include two dummy variables in the conditional volatility equation. The dummy variable $D O$ equals 1 for the first 15 -minute period of the trading day and 0 otherwise. $D C$ equals 1 for the last 15 -minute period of the trading day and 0 otherwise.

The model testing the impact of the information flow on price volatility during the continuous market can be presented as follows ${ }^{5}$ :

$r_{t}=\phi_{0} r_{t-1}+\phi_{1} \varepsilon_{t-1}+\varepsilon_{t}$

$\log \left(h_{t}\right)=C+\gamma Z_{t-1}+\alpha\left(\left|Z_{t-1}\right|-\sqrt{\frac{2}{\pi}}\right)+\beta \log \left(h_{t-1}\right)+\lambda N_{t}+\theta_{1} D O+\theta_{2} D C$

We compute stock price returns with the logarithm formula:

$r_{t}=100 \ln \left(P_{t} / P_{t-1}\right)$

Where $P_{t}$ is the stock price at the end of each 15-minute period t.

$N_{t}$ represents the number of news items published by Reuters during each 15-minute period t.

The impact of overnight information flow on open-to-close volatility is tested by the following model:

$\bar{r}=\bar{\phi}_{0} \bar{r}_{t-1}+\bar{\phi}_{1} \bar{\varepsilon}_{t-1}+\bar{\varepsilon}_{t}$

\footnotetext{
${ }^{4}$ This routine is used to compute the quasi-maximum likelihood (QML) covariances and standard errors using the methods described by Bollerslev and Wooldridge (1992). The parameter estimates will be unchanged, only the estimated covariance matrix and p-values will be altered.

${ }^{5}$ Stock returns are modelled by a moving average process of order 1 to account for autocorrelation.
} 


$$
\begin{aligned}
& \log \left(\bar{h}_{t}\right)=\bar{C}+\overline{\gamma Z}_{t-1}+\bar{\alpha}\left(\left|\bar{Z}_{t-1}\right|-\sqrt{\frac{2}{\pi}}\right)+\bar{\beta} \log \left(\bar{h}_{t-1}\right)+\bar{\lambda} \bar{N}_{t} \\
& \bar{r}_{t}=100 \ln \left(\bar{P}_{t} / \bar{P}_{t-1}\right)
\end{aligned}
$$

Where $\bar{P}_{t}$ is the opening price of the day $\mathrm{t}$ and $\bar{P}_{t-1}$ is the closing price of the day $\mathrm{t}-1$.

$\bar{N}_{t}$ is the number of overnight news items published by Reuters.

\subsection{Trading volume}

The mixture distribution hypothesis provides evidence that the information flow impacts stock prices as well as trading volume. Event studies conducted around public announcements confirm this intuition. Indeed, these studies show that news releases are accompanied by a significant price variation and an abnormal high trading volume (Louhichi, 2008). Blume, Easley and O'Hara (1994) investigate the usefulness of trading volume for technical analysis. The authors highlight the informational role of the volume statistic. The authors conclude that trading volume and price changes have distinct and complementary informational roles. They recommend the use of both trading volume and returns for financial forecasting.

A simple way to examine the relationship between trading volume and the information flow is to regress the trading volume against the number of news items published by Reuters during each interval t. The model testing the impact of the information flow on trading volume during the continuous market can be presented as follows:

$$
\log \left(V_{t}\right)=C+\lambda N_{t}+\theta_{1} D O+\theta_{2} D C+\varepsilon_{t}
$$

$N_{t}$ is the number of news items published by Reuters during each 15 -minute period t.

$V_{t}$ is the trading volume during each 15 -minute period $\mathrm{t}$.

The impact of overnight information flow on opening trading volume is tested by the following model:

$$
\log \left(\bar{V}_{t}\right)=\bar{C}+\bar{\lambda} \bar{N}_{t}+\bar{\varepsilon}_{t}
$$

$\bar{N}_{t}$ is the number of overnight news items.

$\bar{V}_{t}$ is the opening trading volume.

\subsection{Data}

The aim of this paper is to shed light on the relationship between the public information flow and market activity using high-frequency data from Euronext Paris. The study requires two types of data: a proxy for public information arrival and quantitative information about stocks. To measure the rate of information flow, we use the proxy proposed bay Berry and Howe (1994). We quantify the information flow as the number of news items published by Reuters during each period of 15 -minute. Reuters is used by most financial operators in Euronext and has the advantage of providing the time of announcement to the nearest minute. Furthermore, our proxy is used in several papers (Berry and Howe (1994), Andersen and Bollerslev (1998), Melvin and Yin (2000)) related to our study. This allows us to compare our results to those obtained in markets characterized by a different structure that of the French market. From the Reuters database, we collect all news concerning companies pertaining to the 
CAC40 index and recorded from January through December 2001 in the category "Corporate News". This section contains all information which affects only a specific firm (firm specific news).

Quantitative information concerns intraday data about trades, execution date and time, price and trading volume. This information is obtained from Euronext database and covers the period from January 2001 to December 2001. During this period, we divide the trading day into thirty four 15-minute intervals.

Our sample concerns all shares pertaining to the CAC40 Index, which is the main benchmark for Euronext Paris. The composition of the CAC40 was marked by two changes during 2001. The first occurred on May 4 when "Orange" replaced "Equant". Similarly, on August 8 "Valeo" replaced "Vivendi Environment". Finally, the sample consists of 41 companies rather than 42 because we don't have all necessary information about the firm "Bouygues".

\section{RESULTS}

\subsection{Public information arrival and conditional volatility}

The results of estimating the EGARCH model during the continuous market are summarized in Table 1. We notice that the EGARCH coefficients ( $\alpha$ and $\beta$ ) are significant except for the asymmetric effects $(\gamma)$. The coefficient $\beta$ is always less than 1 which confirms the stationarity of the model. Moreover, the introduction of the dummy variables is justified since their coefficients are generally statistically significant. Finally, Table 1 allows us to shed light on the impact of the information flow on the price volatility. The coefficient of the informational variable $N_{t}$ is significantly positive for 11 out of the $40^{6}$ firms of our sample, which represents $27.5 \%$. These findings are statistically significant because, with a 95\% confidence interval, Type I errors expect to find 2 coefficients in each set of 40 that are significant by chance. Our results are out of this range, which show that they are robust.

Table 2 summarizes the estimation results of Eq. (4). This table confirms the results detailed above. Indeed, the EGARCH coefficients ( $\alpha$ and $\beta$ ) are generally significant and ensuring the stationarity of the model. The coefficient $\lambda$ is significantly positive for 18 out of the $38^{7}$ firms of our sample, which represents $47.5 \%$. These findings show that high opening volatility is explained by both informational causes (accumulation of public information during the closing period) and microstructure factors related to the opening protocol (the opening effect documented by Jain and Joh (1985), Wood et al. (1985) and Blau et al. (2009) among others).

We have also tested our models for the market index (CAC40). Table 1 and Table 2 show that the coefficient of the informational variable $\left(N_{t}\right)$ is not significant during the continuous market as well as for overnight announcements. In conclusion, our findings show that there is a moderate positive relationship between the number of news releases recorded by Reuters and stock price volatility. Our results are different from those found by Berry and Howe (1994) on the U.S. market and those obtained by Kalev et al. (2004) on the Australian market. The first authors highlight an insignificant relationship between the number of headlines published by Reuters and price volatility. Kalev et al. (2004) show that stock price volatility increases significantly around news releases. The moderate relationship between volatility and our informational proxy can be explained by several reasons. Indeed, we have taken into account all the news published by Reuters. This means that our sample can contain some announcements which were anticipated by the market. Therefore, this category of news has no information content and has been already integrated into prices. Moreover, several significant price changes occur without informational cause. The crash of October 1987 and the studies of Fair (2002) and Culter, Poterba and Summers (1989) confirm this hypothesis.

\footnotetext{
${ }^{6}$ The model didn't converge for the firm Peugeot.

${ }^{7}$ The model didn't converge for 3 firms: Axa, Peugeot and Sodexho.
} 
Table 1: the intraday relation between volatility and news releases during the continuous market

\begin{tabular}{|c|c|c|c|c|c|c|c|c|c|}
\hline Titre & $\phi_{0}$ & $\phi_{1}$ & $C$ & $\gamma$ & $\bar{\alpha}$ & $\beta$ & $\lambda$ & $\theta_{1}$ & $\theta_{2}$ \\
\hline Accor & $\begin{array}{l}0.024 \\
(0.85)\end{array}$ & $\begin{array}{l}-0.119 \\
(0.37)\end{array}$ & $\begin{array}{l}-2.298 \\
(0.00)\end{array}$ & $\begin{array}{l}-0.014 \\
(0.58)\end{array}$ & $\begin{array}{l}0.445 \\
(0.00)\end{array}$ & $\begin{array}{l}0.824 \\
(0.00)\end{array}$ & $\begin{array}{l}0.706 \\
(0.00)\end{array}$ & $\begin{array}{l}1.288 \\
(0.00)\end{array}$ & $\begin{array}{l}0.534 \\
(0.00)\end{array}$ \\
\hline AGF & $\begin{array}{l}0.180 \\
(0.00)\end{array}$ & $\begin{array}{l}-0.358 \\
(0.00)\end{array}$ & $\begin{array}{l}-2.793 \\
(0.00)\end{array}$ & $\begin{array}{l}-0.003 \\
(0.90)\end{array}$ & $\begin{array}{l}0.423 \\
(0.00)\end{array}$ & $\begin{array}{l}0.779 \\
(0.00)\end{array}$ & $\begin{array}{l}0.639 \\
(0.06)\end{array}$ & $\begin{array}{l}0.848 \\
(0.00)\end{array}$ & $\begin{array}{l}0.761 \\
(0.00)\end{array}$ \\
\hline Air Liquide & $\begin{array}{l}0.257 \\
(0.00)\end{array}$ & $\begin{array}{l}-0.445 \\
(0.00)\end{array}$ & $\begin{array}{l}-1.633 \\
(0.00)\end{array}$ & $\begin{array}{l}0.050 \\
(0.19)\end{array}$ & $\begin{array}{l}0.328 \\
(0.00)\end{array}$ & $\begin{array}{l}0.879 \\
(0.00)\end{array}$ & $\begin{array}{l}-0.021 \\
(0.94)\end{array}$ & $\begin{array}{l}0.723 \\
(0.00)\end{array}$ & $\begin{array}{l}0.612 \\
(0.00)\end{array}$ \\
\hline Alcatel & $\begin{array}{l}0.255 \\
(0.03)\end{array}$ & $\begin{array}{l}-0.283 \\
(0.02)\end{array}$ & $\begin{array}{l}-4.166 \\
(0.00)\end{array}$ & $\begin{array}{l}-0.031 \\
(0.09)\end{array}$ & $\begin{array}{l}0.398 \\
(0.00)\end{array}$ & $\begin{array}{l}0.622 \\
(0.00)\end{array}$ & $\begin{array}{l}0.155 \\
(0.18) \\
\end{array}$ & $\begin{array}{l}2.710 \\
(0.00)\end{array}$ & $\begin{array}{l}-0.043 \\
(0.68) \\
\end{array}$ \\
\hline Alstom & $\begin{array}{l}0.004 \\
(0.98)\end{array}$ & $\begin{array}{l}-0.059 \\
(0.82)\end{array}$ & $\begin{array}{c}-1.878 \\
(0.00)\end{array}$ & $\begin{array}{c}-0.042 \\
(0.10)\end{array}$ & $\begin{array}{l}0.459 \\
(0.00)\end{array}$ & $\begin{array}{l}0.860 \\
(0.00)\end{array}$ & $\begin{array}{l}0.532 \\
(0.00)\end{array}$ & $\begin{array}{l}1.415 \\
(0.00)\end{array}$ & $\begin{array}{l}0.305 \\
(0.02)\end{array}$ \\
\hline Aventis & $\begin{array}{l}0.152 \\
(0.283)\end{array}$ & $\begin{array}{l}-0.222 \\
(0.11)\end{array}$ & $\begin{array}{l}-2.440 \\
(0.00)\end{array}$ & $\begin{array}{l}-0.035 \\
(0.05)\end{array}$ & $\begin{array}{l}0.372 \\
(0.00)\end{array}$ & $\begin{array}{l}0.814 \\
(0.00)\end{array}$ & $\begin{array}{l}0.144 \\
(0.44)\end{array}$ & $\begin{array}{l}1.354 \\
(0.00)\end{array}$ & $\begin{array}{l}0.401 \\
(0.00)\end{array}$ \\
\hline Axa & $\begin{array}{l}0.861 \\
(0.01)\end{array}$ & $\begin{array}{l}-0.848 \\
(0.01)\end{array}$ & $\begin{array}{l}-4.026 \\
(0.92)\end{array}$ & $\begin{array}{l}0.127 \\
(0.93)\end{array}$ & $\begin{array}{l}0.253 \\
(0.77)\end{array}$ & $\begin{array}{l}0.618 \\
(0.88)\end{array}$ & $\begin{array}{l}-0.049 \\
(0.94)\end{array}$ & $\begin{array}{l}1.636 \\
(0.96)\end{array}$ & $\begin{array}{l}0.107 \\
(0.97)\end{array}$ \\
\hline Bnp Paribas & $\begin{array}{l}0.070 \\
(0.49) \\
\end{array}$ & $\begin{array}{l}-0.189 \\
(0.06) \\
\end{array}$ & $\begin{array}{l}-2.795 \\
(0.00) \\
\end{array}$ & $\begin{array}{l}-0.001 \\
(0.95) \\
\end{array}$ & $\begin{array}{l}0.410 \\
(0.00) \\
\end{array}$ & $\begin{array}{l}0.795 \\
(0.00) \\
\end{array}$ & $\begin{array}{l}0.231 \\
(0.21) \\
\end{array}$ & $\begin{array}{l}1.595 \\
(0.00) \\
\end{array}$ & $\begin{array}{l}0.363 \\
(0.00) \\
\end{array}$ \\
\hline Cap Gemini & $\begin{array}{l}-0.138 \\
(0.35)\end{array}$ & $\begin{array}{l}0.073 \\
(0.63)\end{array}$ & $\begin{array}{l}-4.512 \\
(0.00)\end{array}$ & $\begin{array}{l}0.012 \\
(0.60)\end{array}$ & $\begin{array}{l}0.454 \\
(0.00)\end{array}$ & $\begin{array}{l}0.604 \\
(0.00)\end{array}$ & $\begin{array}{l}0.826 \\
(0.05)\end{array}$ & $\begin{array}{l}2.542 \\
(0.00)\end{array}$ & $\begin{array}{l}0.120 \\
(0.24)\end{array}$ \\
\hline Carrefour & $\begin{array}{l}0.248 \\
(0.00)\end{array}$ & $\begin{array}{l}-0.385 \\
(0.00)\end{array}$ & $\begin{array}{l}-1.700 \\
(0.00)\end{array}$ & $\begin{array}{l}0.000 \\
(0.99)\end{array}$ & $\begin{array}{l}0.343 \\
(0.00)\end{array}$ & $\begin{array}{l}0.875 \\
(0.00)\end{array}$ & $\begin{array}{l}0.426 \\
(0.13)\end{array}$ & $\begin{array}{l}1.064 \\
(0.00)\end{array}$ & $\begin{array}{l}0.391 \\
(0.00)\end{array}$ \\
\hline Casino & $\begin{array}{l}0.163 \\
(0.00)\end{array}$ & $\begin{array}{l}-0.405 \\
(0.00)\end{array}$ & $\begin{array}{l}-2.704 \\
(0.00)\end{array}$ & $\begin{array}{l}-0.026 \\
(0.15)\end{array}$ & $\begin{array}{l}0.360 \\
(0.00)\end{array}$ & $\begin{array}{l}0.791 \\
(0.00)\end{array}$ & $\begin{array}{l}0.044 \\
(0.86)\end{array}$ & $\begin{array}{l}0.900 \\
(0.00)\end{array}$ & $\begin{array}{l}0.616 \\
(0.00)\end{array}$ \\
\hline Crédit L & $\begin{array}{l}0.211 \\
(0.00)\end{array}$ & $\begin{array}{c}-0.369 \\
(0.00)\end{array}$ & $\begin{array}{l}-3.076 \\
(0.00)\end{array}$ & $\begin{array}{l}0.062 \\
(0.00)\end{array}$ & $\begin{array}{l}0.379 \\
(0.00)\end{array}$ & $\begin{array}{l}0.761 \\
(0.00)\end{array}$ & $\begin{array}{l}-0.161 \\
(0.48)\end{array}$ & $\begin{array}{l}1.311 \\
(0.00)\end{array}$ & $\begin{array}{l}0.707 \\
(0.00)\end{array}$ \\
\hline Dassault & $\begin{array}{l}0.033 \\
(0.78)\end{array}$ & $\begin{array}{l}-0.102 \\
(0.41)\end{array}$ & $\begin{array}{l}-3.708 \\
(0.00)\end{array}$ & $\begin{array}{l}0.032 \\
(0.12)\end{array}$ & $\begin{array}{l}0.417 \\
(0.00)\end{array}$ & $\begin{array}{l}0.669 \\
(0.00)\end{array}$ & $\begin{array}{l}0.061 \\
(0.79)\end{array}$ & $\begin{array}{l}2.015 \\
(0.00)\end{array}$ & $\begin{array}{l}0.310 \\
(0.00)\end{array}$ \\
\hline Dexia & $\begin{array}{l}0.090 \\
(0.65)\end{array}$ & $\begin{array}{l}0.379 \\
(0.00)\end{array}$ & $\begin{array}{l}-10.254 \\
(0.00)\end{array}$ & $\begin{array}{l}-0.409 \\
(0.24)\end{array}$ & $\begin{array}{l}0.955 \\
(0.14)\end{array}$ & $\begin{array}{l}0.124 \\
(0.40)\end{array}$ & $\begin{array}{l}0.584 \\
(0.03)\end{array}$ & $\begin{array}{l}2.366 \\
0.065\end{array}$ & $\begin{array}{l}-1.378 \\
(0.04)\end{array}$ \\
\hline Eads & $\begin{array}{l}0.914 \\
(0.00)\end{array}$ & $\begin{array}{l}-0.950 \\
(0.00)\end{array}$ & $\begin{array}{l}-2.614 \\
(0.00)\end{array}$ & $\begin{array}{l}-0.043 \\
(0.27)\end{array}$ & $\begin{array}{l}0.518 \\
(0.00)\end{array}$ & $\begin{array}{l}0.792 \\
(0.00)\end{array}$ & $\begin{array}{l}0.083 \\
(0.63)\end{array}$ & $\begin{array}{l}1.436 \\
(0.00)\end{array}$ & $\begin{array}{l}0.442 \\
(0.00)\end{array}$ \\
\hline Equant & $\begin{array}{l}0.215 \\
(0.59)\end{array}$ & $\begin{array}{l}-0.195 \\
(0.62)\end{array}$ & $\begin{array}{c}-1.062 \\
(0.87)\end{array}$ & $\begin{array}{l}0.008 \\
(0.97)\end{array}$ & $\begin{array}{l}0.022 \\
(0.62)\end{array}$ & $\begin{array}{l}0.894 \\
(0.19)\end{array}$ & $\begin{array}{l}-0.068 \\
(0.99)\end{array}$ & $\begin{array}{l}0.174 \\
(0.96)\end{array}$ & $\begin{array}{l}0.042 \\
(0.92)\end{array}$ \\
\hline France Télécom & $\begin{array}{l}-0.831 \\
(0.00)\end{array}$ & $\begin{array}{l}0.816 \\
(0.00)\end{array}$ & $\begin{array}{l}-3.119 \\
(0.00)\end{array}$ & $\begin{array}{l}0.003 \\
(0.87)\end{array}$ & $\begin{array}{l}0.371 \\
(0.00)\end{array}$ & $\begin{array}{l}0.736 \\
(0.00)\end{array}$ & $\begin{array}{l}-0.007 \\
(0.95)\end{array}$ & $\begin{array}{l}2.245 \\
(0.00)\end{array}$ & $\begin{array}{l}0.123 \\
(0.27)\end{array}$ \\
\hline Danone & $\begin{array}{l}0.197 \\
(0.00)\end{array}$ & $\begin{array}{l}-0.341 \\
(0.00)\end{array}$ & $\begin{array}{l}-3.023 \\
(0.00)\end{array}$ & $\begin{array}{l}-0.030 \\
(0.11)\end{array}$ & $\begin{array}{l}0.346 \\
(0.00)\end{array}$ & $\begin{array}{l}0.763 \\
(0.00)\end{array}$ & $\begin{array}{l}0.115 \\
(0.42)\end{array}$ & $\begin{array}{l}1.500 \\
(0.00)\end{array}$ & $\begin{array}{l}0.626 \\
(0.00)\end{array}$ \\
\hline Lafarge & $\begin{array}{l}0.062 \\
(0.47)\end{array}$ & $\begin{array}{c}-0.203 \\
(0.01)\end{array}$ & $\begin{array}{l}-2.901 \\
(0.00)\end{array}$ & $\begin{array}{l}-0.017 \\
(0.34)\end{array}$ & $\begin{array}{l}0.352 \\
(0.00)\end{array}$ & $\begin{array}{l}0.772 \\
(0.00)\end{array}$ & $\begin{array}{l}0.288 \\
(0.28)\end{array}$ & $\begin{array}{l}1.222 \\
(0.00)\end{array}$ & $\begin{array}{l}0.583 \\
(0.00)\end{array}$ \\
\hline Lagardère & $\begin{array}{l}-0.059 \\
(0.66)\end{array}$ & $\begin{array}{l}-0.038 \\
(0.78)\end{array}$ & $\begin{array}{l}-3.390 \\
(0.00)\end{array}$ & $\begin{array}{l}-0.028 \\
(0.17)\end{array}$ & $\begin{array}{l}0.417 \\
(0.00)\end{array}$ & $\begin{array}{l}0.715 \\
(0.00)\end{array}$ & $\begin{array}{l}0.411 \\
(0.01)\end{array}$ & $\begin{array}{l}1.116 \\
(0.00)\end{array}$ & $\begin{array}{l}1.122 \\
(0.00)\end{array}$ \\
\hline L'oréal & $\begin{array}{l}0.283 \\
(0.00)\end{array}$ & $\begin{array}{l}-0.383 \\
(0.00)\end{array}$ & $\begin{array}{l}-2.797 \\
(0.00)\end{array}$ & $\begin{array}{l}0.002 \\
(0.91)\end{array}$ & $\begin{array}{l}0.333 \\
(0.00)\end{array}$ & $\begin{array}{l}0.770 \\
(0.00)\end{array}$ & $\begin{array}{l}0.517 \\
(0.20)\end{array}$ & $\begin{array}{l}0.746 \\
(0.00)\end{array}$ & $\begin{array}{l}0.799 \\
(0.00)\end{array}$ \\
\hline LVMH & $\begin{array}{l}-0.159 \\
(0.26)\end{array}$ & $\begin{array}{l}0.097 \\
(0.49)\end{array}$ & $\begin{array}{l}-2.942 \\
(0.00)\end{array}$ & $\begin{array}{l}0.003 \\
(0.86)\end{array}$ & $\begin{array}{l}0.411 \\
(0.00)\end{array}$ & $\begin{array}{l}0.760 \\
(0.00)\end{array}$ & $\begin{array}{l}0.514 \\
(0.01)\end{array}$ & $\begin{array}{l}1.541 \\
(0.00)\end{array}$ & $\begin{array}{l}0.589 \\
(0.00)\end{array}$ \\
\hline Michelin & $\begin{array}{l}0.217 \\
(0.00)\end{array}$ & $\begin{array}{l}-0.392 \\
(0.00)\end{array}$ & $\begin{array}{l}-3.462 \\
(0.00)\end{array}$ & $\begin{array}{l}0.005 \\
(0.84)\end{array}$ & $\begin{array}{l}0.463 \\
(0.00)\end{array}$ & $\begin{array}{l}0.717 \\
(0.00)\end{array}$ & $\begin{array}{l}0.902 \\
(0.06)\end{array}$ & $\begin{array}{l}1.594 \\
(0.00)\end{array}$ & $\begin{array}{l}0.385 \\
(0.00)\end{array}$ \\
\hline
\end{tabular}


Table 1 (continued)

\begin{tabular}{|c|c|c|c|c|c|c|c|c|c|}
\hline Titre & $\phi_{0}$ & $\phi_{1}$ & $C$ & $\gamma$ & $\alpha$ & $\beta$ & $\lambda$ & $\theta_{1}$ & $\theta 2$ \\
\hline Orange & $\begin{array}{l}0.080 \\
(0.34)\end{array}$ & $\begin{array}{l}-0.187 \\
(0.02)\end{array}$ & $\begin{array}{l}-2.554 \\
(0.00)\end{array}$ & $\begin{array}{l}0.013 \\
(0.50)\end{array}$ & $\begin{array}{l}0.341 \\
(0.00)\end{array}$ & $\begin{array}{l}0.795 \\
(0.00)\end{array}$ & $\begin{array}{l}0.074 \\
(0.73)\end{array}$ & $\begin{array}{l}1.563 \\
(0.00)\end{array}$ & $\begin{array}{l}0.282 \\
(0.02)\end{array}$ \\
\hline Peugeot & - & - & - & - & - & - & - & - & - \\
\hline Pinault-PR & $\begin{array}{l}0.003 \\
(0.97)\end{array}$ & $\begin{array}{l}-0.136 \\
(0.18)\end{array}$ & $\begin{array}{l}-2.234 \\
(0.00)\end{array}$ & $\begin{array}{l}-0.020 \\
(0.24)\end{array}$ & $\begin{array}{l}0.385 \\
(0.00)\end{array}$ & $\begin{array}{l}0.830 \\
(0.00)\end{array}$ & $\begin{array}{l}-0.174 \\
(0.33)\end{array}$ & $\begin{array}{l}1.147 \\
(0.00)\end{array}$ & $\begin{array}{l}0.546 \\
(0.00)\end{array}$ \\
\hline Renault & $\begin{array}{l}0.127 \\
(0.25)\end{array}$ & $\begin{array}{l}-0.228 \\
(0.03)\end{array}$ & $\begin{array}{l}-2.462 \\
(0.00)\end{array}$ & $\begin{array}{l}-0.032 \\
(0.16)\end{array}$ & $\begin{array}{l}0.416 \\
(0.00)\end{array}$ & $\begin{array}{l}0.806 \\
(0.00)\end{array}$ & $\begin{array}{l}0.280 \\
(0.14)\end{array}$ & $\begin{array}{l}1.051 \\
(0.00)\end{array}$ & $\begin{array}{l}0.805 \\
(0.00)\end{array}$ \\
\hline Saint-Gobain & $\begin{array}{l}0.168 \\
(0.04)\end{array}$ & $\begin{array}{l}-0.303 \\
(0.00)\end{array}$ & $\begin{array}{l}-2.462 \\
(0.00)\end{array}$ & $\begin{array}{l}0.002 \\
(0.90)\end{array}$ & $\begin{array}{l}0.380 \\
(0.00)\end{array}$ & $\begin{array}{l}0.811 \\
(0.00)\end{array}$ & $\begin{array}{l}-0.145 \\
(0.53)\end{array}$ & $\begin{array}{l}0.916 \\
(0.00)\end{array}$ & $\begin{array}{l}0.838 \\
(0.00)\end{array}$ \\
\hline Sanofi Synthelabo & $\begin{array}{l}0.185 \\
(0.04)\end{array}$ & $\begin{array}{l}-0.275 \\
(0.00)\end{array}$ & $\begin{array}{l}-3.180 \\
(0.00)\end{array}$ & $\begin{array}{l}-0.025 \\
(0.46)\end{array}$ & $\begin{array}{l}0.369 \\
(0.00)\end{array}$ & $\begin{array}{l}0.744 \\
(0.00)\end{array}$ & $\begin{array}{l}0.067 \\
(0.80)\end{array}$ & $\begin{array}{l}1.149 \\
(0.00)\end{array}$ & $\begin{array}{l}0.636 \\
(0.00)\end{array}$ \\
\hline Schneider & $\begin{array}{l}0.256 \\
(0.64)\end{array}$ & $\begin{array}{l}-0.230 \\
(0.67)\end{array}$ & $\begin{array}{l}-1.457 \\
(0.97)\end{array}$ & $\begin{array}{l}0.005 \\
(0.99)\end{array}$ & $\begin{array}{l}0.008 \\
(0.98)\end{array}$ & $\begin{array}{l}0.860 \\
(0.85)\end{array}$ & $\begin{array}{l}-0.011 \\
(0.99)\end{array}$ & $\begin{array}{l}0.030 \\
(0.99)\end{array}$ & $\begin{array}{l}0.130 \\
(0.87)\end{array}$ \\
\hline Sociéte Générale & $\begin{array}{l}-0.127 \\
(0.38)\end{array}$ & $\begin{array}{l}0.030 \\
(0.83)\end{array}$ & $\begin{array}{l}-2.468 \\
(0.00)\end{array}$ & $\begin{array}{l}-0.022 \\
(0.25)\end{array}$ & $\begin{array}{l}0.367 \\
(0.00)\end{array}$ & $\begin{array}{l}0.806 \\
(0.00)\end{array}$ & $\begin{array}{l}-0.023 \\
(0.94)\end{array}$ & $\begin{array}{l}1.220 \\
(0.00)\end{array}$ & $\begin{array}{l}0.551 \\
(0.00)\end{array}$ \\
\hline Sodexho & $\begin{array}{l}0.347 \\
(0.00)\end{array}$ & $\begin{array}{l}-0.484 \\
(0.00)\end{array}$ & $\begin{array}{l}-6.534 \\
(0.59)\end{array}$ & $\begin{array}{l}0.036 \\
(0.97)\end{array}$ & $\begin{array}{l}0.408 \\
(0.72)\end{array}$ & $\begin{array}{l}0.424 \\
(0.69)\end{array}$ & $\begin{array}{l}0.424 \\
(0.26)\end{array}$ & $\begin{array}{l}2.643 \\
(0.87)\end{array}$ & $\begin{array}{l}1.654 \\
(0.00)\end{array}$ \\
\hline ST-Microelectronics & $\begin{array}{l}0.548 \\
(0.00) \\
\end{array}$ & $\begin{array}{l}-0.504 \\
(0.00)\end{array}$ & $\begin{array}{l}-3.013 \\
(0.11) \\
\end{array}$ & $\begin{array}{l}0.052 \\
(0.74) \\
\end{array}$ & $\begin{array}{l}0.069 \\
(0.69) \\
\end{array}$ & $\begin{array}{l}0.715 \\
(0.00) \\
\end{array}$ & $\begin{array}{l}-0.243 \\
(0.58) \\
\end{array}$ & $\begin{array}{l}0.695 \\
(0.59) \\
\end{array}$ & $\begin{array}{l}-0.232 \\
(0.14)\end{array}$ \\
\hline Suez Lyon.Des Eaux & $\begin{array}{l}0.007 \\
(0.95)\end{array}$ & $\begin{array}{l}-0.142 \\
(0.30)\end{array}$ & $\begin{array}{l}-4.325 \\
(0.00)\end{array}$ & $\begin{array}{l}-0.124 \\
(0.12)\end{array}$ & $\begin{array}{l}0.657 \\
(0.00)\end{array}$ & $\begin{array}{l}0.656 \\
(0.00)\end{array}$ & $\begin{array}{l}0.450 \\
(0.13)\end{array}$ & $\begin{array}{l}3.032 \\
(0.00)\end{array}$ & $\begin{array}{l}-0.826 \\
(0.36)\end{array}$ \\
\hline Tf1 & $\begin{array}{l}0.092 \\
(0.49)\end{array}$ & $\begin{array}{l}-0.114 \\
(0.40)\end{array}$ & $\begin{array}{l}-2.542 \\
(0.00)\end{array}$ & $\begin{array}{l}-0.009 \\
(0.72)\end{array}$ & $\begin{array}{l}0.388 \\
(0.00)\end{array}$ & $\begin{array}{l}0.786 \\
(0.00)\end{array}$ & $\begin{array}{l}0.609 \\
(0.09)\end{array}$ & $\begin{array}{l}1.564 \\
(0.00)\end{array}$ & $\begin{array}{l}0.563 \\
(0.00)\end{array}$ \\
\hline Thales & $\begin{array}{l}0.168 \\
(0.16)\end{array}$ & $\begin{array}{l}-0.268 \\
(0.02)\end{array}$ & $\begin{array}{l}-4.008 \\
(0.00)\end{array}$ & $\begin{array}{l}-0.022 \\
(0.26)\end{array}$ & $\begin{array}{l}0.412 \\
(0.00)\end{array}$ & $\begin{array}{l}0.657 \\
(0.00)\end{array}$ & $\begin{array}{l}0.979 \\
(0.03)\end{array}$ & $\begin{array}{l}1.197 \\
(0.00)\end{array}$ & $\begin{array}{l}0.432 \\
(0.00)\end{array}$ \\
\hline Thomson & $\begin{array}{l}-0.208 \\
(0.09)\end{array}$ & $\begin{array}{l}0.156 \\
(0.21)\end{array}$ & $\begin{array}{l}-3.105 \\
(0.00)\end{array}$ & $\begin{array}{l}-0.011 \\
(0.66)\end{array}$ & $\begin{array}{l}0.382 \\
(0.00)\end{array}$ & $\begin{array}{l}0.729 \\
(0.00)\end{array}$ & $\begin{array}{l}1.175 \\
(0.02)\end{array}$ & $\begin{array}{l}1.930 \\
(0.00)\end{array}$ & $\begin{array}{l}0.536 \\
(0.00)\end{array}$ \\
\hline Total Fina Elf & $\begin{array}{l}-0.280 \\
(0.08)\end{array}$ & $\begin{array}{l}0.210 \\
(0.20)\end{array}$ & $\begin{array}{l}-3.095 \\
(0.00)\end{array}$ & $\begin{array}{l}-0.016 \\
(0.35)\end{array}$ & $\begin{array}{l}0.341 \\
(0.00)\end{array}$ & $\begin{array}{l}0.759 \\
(0.00)\end{array}$ & $\begin{array}{l}0.095 \\
(0.53)\end{array}$ & $\begin{array}{l}1.238 \\
(0.00)\end{array}$ & $\begin{array}{l}0.801 \\
(0.00)\end{array}$ \\
\hline Valeo & $\begin{array}{l}0.149 \\
(0.04)\end{array}$ & $\begin{array}{l}-0.336 \\
(0.00)\end{array}$ & $\begin{array}{l}-3.081 \\
(0.00) \\
\end{array}$ & $\begin{array}{l}0.025 \\
(0.27) \\
\end{array}$ & $\begin{array}{l}0.374 \\
(0.00) \\
\end{array}$ & $\begin{array}{l}0.746 \\
(0.00) \\
\end{array}$ & $\begin{array}{l}0.290 \\
(0.41)\end{array}$ & $\begin{array}{l}1.012 \\
(0.00)\end{array}$ & $\begin{array}{l}1.053 \\
(0.00) \\
\end{array}$ \\
\hline Vivendi Environement & $\begin{array}{l}0.398 \\
(0.00) \\
\end{array}$ & $\begin{array}{l}-0.542 \\
(0.00)\end{array}$ & $\begin{array}{l}-4.139 \\
(0.00) \\
\end{array}$ & $\begin{array}{l}-0.006 \\
(0.82) \\
\end{array}$ & $\begin{array}{l}0.470 \\
(0.00) \\
\end{array}$ & $\begin{array}{l}0.671 \\
(0.00) \\
\end{array}$ & $\begin{array}{l}-0.175 \\
(0.50) \\
\end{array}$ & $\begin{array}{l}1.412 \\
(0.00)\end{array}$ & $\begin{array}{l}0.699 \\
(0.00) \\
\end{array}$ \\
\hline Vivendi Universal & $\begin{array}{l}-0.100 \\
(0.00) \\
\end{array}$ & $\begin{array}{l}0.033 \\
(0.26) \\
\end{array}$ & $\begin{array}{l}-2.368 \\
(0.00) \\
\end{array}$ & $\begin{array}{l}-0.029 \\
(0.14) \\
\end{array}$ & $\begin{array}{l}0.406 \\
(0.00) \\
\end{array}$ & $\begin{array}{l}0.819 \\
(0.00) \\
\end{array}$ & $\begin{array}{l}0.183 \\
(0.29) \\
\end{array}$ & $\begin{array}{l}1.610 \\
(0.00) \\
\end{array}$ & $\begin{array}{l}0.521 \\
(0.00) \\
\end{array}$ \\
\hline CAC40 & $\begin{array}{l}0.394 \\
(0.19)\end{array}$ & $\begin{array}{l}-0.376 \\
(0.22)\end{array}$ & $\begin{array}{l}-3.286 \\
(0.00)\end{array}$ & $\begin{array}{l}-0.011 \\
(0.54)\end{array}$ & $\begin{array}{l}0.411 \\
(0.00)\end{array}$ & $\begin{array}{l}0.762 \\
(0.00)\end{array}$ & $\begin{array}{l}-0.090 \\
(0.90)\end{array}$ & $\begin{array}{l}2.296 \\
(0.00)\end{array}$ & $\begin{array}{l}0.155 \\
(0.28)\end{array}$ \\
\hline
\end{tabular}

Table 1 - This table presents the results of the estimation of model (3) which tests the intraday relation between volatility and news releases during the continuous market. Volatility is estimated with the EGARCH model proposed by Nelson (1991). $r_{t}$ and $h_{t}$ are respectively the return and the conditional volatility during the 15-minute period t. $\mathrm{N}_{\mathrm{t}}$ is the number of news items published by Reuters during each 15 -interval t. DO and DC are two dummy variables accounting respectively for the first 15-minute period of the trading day and for the last 15-minute period. P-values (in parentheses) are calculated using Bollerslev and Wooldrige's (1992) robust standard errors. 
Table 2: the relation between volatility and overnight announcements

\begin{tabular}{|c|c|c|c|c|c|c|c|}
\hline Titre & $\phi_{0}$ & $\phi_{1}$ & $C$ & $\gamma$ & $\alpha$ & $\beta$ & $\lambda$ \\
\hline Accor & $\begin{array}{l}1.001 \\
(0.00)\end{array}$ & $\begin{array}{l}-0.989 \\
(0.00)\end{array}$ & $\begin{array}{l}-0.704 \\
(0.15)\end{array}$ & $\begin{array}{l}0.128 \\
(0.12)\end{array}$ & $\begin{array}{l}0.212 \\
(0.00)\end{array}$ & $\begin{array}{l}0.936 \\
(0.00)\end{array}$ & $\begin{array}{l}0.088 \\
(0.85)\end{array}$ \\
\hline $\mathrm{AGF}$ & $\begin{array}{l}-0.775 \\
(0.00) \\
\end{array}$ & $\begin{array}{l}0.805 \\
(0.00)\end{array}$ & $\begin{array}{l}-7.495 \\
(0.00)\end{array}$ & $\begin{array}{l}0.187 \\
(0.18)\end{array}$ & $\begin{array}{l}0.747 \\
(0.00)\end{array}$ & $\begin{array}{l}0.244 \\
(0.17)\end{array}$ & $\begin{array}{l}0.114 \\
(0.74)\end{array}$ \\
\hline Air Liquide & $\begin{array}{l}0.986 \\
(0.00) \\
\end{array}$ & $\begin{array}{l}-0.926 \\
(0.00)\end{array}$ & $\begin{array}{l}-8.270 \\
(0.04) \\
\end{array}$ & $\begin{array}{l}0.065 \\
(0.49)\end{array}$ & $\begin{array}{l}0.010 \\
(0.97)\end{array}$ & $\begin{array}{l}0.104 \\
(0.82)\end{array}$ & $\begin{array}{l}-0.356 \\
(0.14) \\
\end{array}$ \\
\hline Alcatel & $\begin{array}{l}-0.490 \\
(0.15) \\
\end{array}$ & $\begin{array}{l}0.490 \\
(0.16)\end{array}$ & $\begin{array}{l}-3.957 \\
(0.22)\end{array}$ & $\begin{array}{l}-0.101 \\
(0.30)\end{array}$ & $\begin{array}{l}0.272 \\
(0.08)\end{array}$ & $\begin{array}{l}0.490 \\
(0.26)\end{array}$ & $\begin{array}{l}0.045 \\
(0.48)\end{array}$ \\
\hline Alstom & $\begin{array}{l}0.928 \\
(0.00)\end{array}$ & $\begin{array}{l}-0.848 \\
(0.00)\end{array}$ & $\begin{array}{l}-0.899 \\
(0.16)\end{array}$ & $\begin{array}{l}-0.037 \\
(0.56)\end{array}$ & $\begin{array}{l}0.179 \\
(0.11)\end{array}$ & $\begin{array}{l}0.918 \\
(0.00)\end{array}$ & $\begin{array}{l}0.386 \\
(0.01)\end{array}$ \\
\hline Aventis & $\begin{array}{l}-0.009 \\
(0.96) \\
\end{array}$ & $\begin{array}{l}-0.016 \\
(0.93)\end{array}$ & $\begin{array}{l}-6.058 \\
(0.06) \\
\end{array}$ & $\begin{array}{l}-0.043 \\
(0.69) \\
\end{array}$ & $\begin{array}{l}0.359 \\
(0.00)\end{array}$ & $\begin{array}{l}0.388 \\
(0.26) \\
\end{array}$ & $\begin{array}{l}0.186 \\
(0.24)\end{array}$ \\
\hline Axa & - & - & - & - & - & - & - \\
\hline Bnp Paribas & $\begin{array}{l}-0.997 \\
(0.00)\end{array}$ & $\begin{array}{l}0.992 \\
(0.00)\end{array}$ & $\begin{array}{l}-2.265 \\
(0.18)\end{array}$ & $\begin{array}{l}0.075 \\
(0.77)\end{array}$ & $\begin{array}{l}0.214 \\
(0.41)\end{array}$ & $\begin{array}{l}0.791 \\
(0.00)\end{array}$ & $\begin{array}{l}0.139 \\
(0.45)\end{array}$ \\
\hline Cap Gemini & $\begin{array}{l}-0.513 \\
(0.01) \\
\end{array}$ & $\begin{array}{l}0.581 \\
(0.00)\end{array}$ & $\begin{array}{l}-14.572 \\
(0.00)\end{array}$ & $\begin{array}{l}-0.027 \\
(0.22)\end{array}$ & $\begin{array}{l}0.447 \\
(0.00)\end{array}$ & $\begin{array}{l}-0.867 \\
(0.00)\end{array}$ & $\begin{array}{l}0.491 \\
(0.00)\end{array}$ \\
\hline Carrefour & $\begin{array}{l}0.395 \\
(0.26)\end{array}$ & $\begin{array}{l}-0.433 \\
(0.22)\end{array}$ & $\begin{array}{l}-8.037 \\
(0.00)\end{array}$ & $\begin{array}{l}-0.150 \\
(0.13)\end{array}$ & $\begin{array}{l}-0.140 \\
(0.58)\end{array}$ & $\begin{array}{l}0.154 \\
(0.55)\end{array}$ & $\begin{array}{l}0.561 \\
(0.00)\end{array}$ \\
\hline Casino & $\begin{array}{l}0.752 \\
(0.00)\end{array}$ & $\begin{array}{l}-0.631 \\
(0.00)\end{array}$ & $\begin{array}{l}-12.603 \\
(0.00)\end{array}$ & $\begin{array}{l}0.105 \\
(0.12)\end{array}$ & $\begin{array}{l}0.587 \\
(0.00)\end{array}$ & $\begin{array}{l}-0.279 \\
(0.00)\end{array}$ & $\begin{array}{l}1.550 \\
(0.00)\end{array}$ \\
\hline Crédit Lyonnais & $\begin{array}{l}0.997 \\
(0.00) \\
\end{array}$ & $\begin{array}{l}-0.989 \\
(0.00) \\
\end{array}$ & $\begin{array}{l}-9.493 \\
(0.18) \\
\end{array}$ & $\begin{array}{l}0.144 \\
(0.13) \\
\end{array}$ & $\begin{array}{l}-0.740 \\
(0.29) \\
\end{array}$ & $\begin{array}{l}-0.084 \\
(0.92)\end{array}$ & $\begin{array}{l}-0.433 \\
(0.07) \\
\end{array}$ \\
\hline Dassault & $\begin{array}{l}-0.522 \\
(0.00)\end{array}$ & $\begin{array}{l}0.710 \\
(0.00)\end{array}$ & $\begin{array}{l}-5.032 \\
(0.15)\end{array}$ & $\begin{array}{l}0.117 \\
(0.27)\end{array}$ & $\begin{array}{l}0.155 \\
(0.24)\end{array}$ & $\begin{array}{l}0.374 \\
(0.40)\end{array}$ & $\begin{array}{l}0.693 \\
(0.03)\end{array}$ \\
\hline Dexia & $\begin{array}{l}0.005 \\
(0.99)\end{array}$ & $\begin{array}{l}0.005 \\
(0.99)\end{array}$ & $\begin{array}{l}-3.859 \\
(0.00)\end{array}$ & $\begin{array}{l}0.010 \\
(0.99)\end{array}$ & $\begin{array}{l}0.010 \\
(0.99)\end{array}$ & $\begin{array}{l}0.010 \\
(0.98)\end{array}$ & $\begin{array}{l}0.000 \\
(1.00)\end{array}$ \\
\hline Eads & $\begin{array}{l}0.858 \\
(0.00)\end{array}$ & $\begin{array}{l}-0.751 \\
(0.00)\end{array}$ & $\begin{array}{l}-2.760 \\
(0.00)\end{array}$ & $\begin{array}{l}0.198 \\
(0.05)\end{array}$ & $\begin{array}{l}0.470 \\
(0.00)\end{array}$ & $\begin{array}{l}0.710 \\
(0.00)\end{array}$ & $\begin{array}{l}0.250 \\
(0.37)\end{array}$ \\
\hline Equant & $\begin{array}{l}-0.328 \\
(0.00)\end{array}$ & $\begin{array}{l}0.549 \\
(0.00)\end{array}$ & $\begin{array}{l}-9.257 \\
(0.00)\end{array}$ & $\begin{array}{l}1.134 \\
(0.00)\end{array}$ & $\begin{array}{l}1.567 \\
(0.00)\end{array}$ & $\begin{array}{l}-0.108 \\
(0.012)\end{array}$ & $\begin{array}{l}0.691 \\
(0.02)\end{array}$ \\
\hline France Télécom & $\begin{array}{l}0.472 \\
(0.02)\end{array}$ & $\begin{array}{l}-0.602 \\
(0.00)\end{array}$ & $\begin{array}{l}-3.297 \\
(0.05)\end{array}$ & $\begin{array}{l}0.113 \\
(0.15)\end{array}$ & $\begin{array}{l}-0.250 \\
(0.07)\end{array}$ & $\begin{array}{l}0.568 \\
(0.00)\end{array}$ & $\begin{array}{l}0.025 \\
(0.74)\end{array}$ \\
\hline Danone & $\begin{array}{l}0.545 \\
(0.00)\end{array}$ & $\begin{array}{l}-0.445 \\
(0.00)\end{array}$ & $\begin{array}{l}-4.143 \\
(0.00)\end{array}$ & $\begin{array}{l}-0.158 \\
(0.01)\end{array}$ & $\begin{array}{l}0.037 \\
(0.76)\end{array}$ & $\begin{array}{l}0.578 \\
(0.00)\end{array}$ & $\begin{array}{l}0.724 \\
(0.00)\end{array}$ \\
\hline Lafarge & $\begin{array}{l}0.997 \\
(0.00)\end{array}$ & $\begin{array}{l}-0.993 \\
(0.00)\end{array}$ & $\begin{array}{l}-17.843 \\
(0.00)\end{array}$ & $\begin{array}{l}0.029 \\
(0.46)\end{array}$ & $\begin{array}{l}0.113 \\
(0.01)\end{array}$ & $\begin{array}{l}-0.938 \\
(0.00)\end{array}$ & $\begin{array}{l}0.435 \\
(0.04)\end{array}$ \\
\hline Lagardère & $\begin{array}{l}-0.298 \\
(0.59)\end{array}$ & $\begin{array}{l}0.421 \\
(0.42)\end{array}$ & $\begin{array}{l}-0.940 \\
(0.16)\end{array}$ & $\begin{array}{l}-0.102 \\
(0.17)\end{array}$ & $\begin{array}{l}0.279 \\
(0.26)\end{array}$ & $\begin{array}{l}0.913 \\
(0.00)\end{array}$ & $\begin{array}{l}0.009 \\
(0.96)\end{array}$ \\
\hline L'oréal & $\begin{array}{l}0.779 \\
(0.00)\end{array}$ & $\begin{array}{l}-0.783 \\
(0.00)\end{array}$ & $\begin{array}{l}-8.549 \\
(0.00)\end{array}$ & $\begin{array}{l}0.016 \\
(0.90)\end{array}$ & $\begin{array}{l}0.153 \\
(0.45)\end{array}$ & $\begin{array}{l}0.075 \\
(0.83)\end{array}$ & $\begin{array}{l}1.405 \\
(0.01)\end{array}$ \\
\hline LVMH & $\begin{array}{l}0.922 \\
(0.00)\end{array}$ & $\begin{array}{l}-0.922 \\
(0.00)\end{array}$ & $\begin{array}{l}-12.753 \\
(0.00)\end{array}$ & $\begin{array}{l}0.189 \\
(0.17)\end{array}$ & $\begin{array}{l}0.416 \\
(0.01)\end{array}$ & $\begin{array}{l}-0.450 \\
(0.00)\end{array}$ & $\begin{array}{l}0.718 \\
(0.00) \\
\end{array}$ \\
\hline Michelin & $\begin{array}{l}0.750 \\
(0.00)\end{array}$ & $\begin{array}{l}-0.564 \\
(0.00)\end{array}$ & $\begin{array}{l}-2.560 \\
(0.00)\end{array}$ & $\begin{array}{l}-0.027 \\
(0.73)\end{array}$ & $\begin{array}{l}0.265 \\
(0.02)\end{array}$ & $\begin{array}{l}0.742 \\
(0.00)\end{array}$ & $\begin{array}{l}0.939 \\
(0.01)\end{array}$ \\
\hline
\end{tabular}


Table 2 (continued)

\begin{tabular}{|c|c|c|c|c|c|c|c|}
\hline Titre & $\phi_{0}$ & $\phi_{1}$ & $C$ & $\gamma$ & $\alpha$ & $\beta$ & $\lambda$ \\
\hline Orange & $\begin{array}{l}-0.807 \\
(0.00)\end{array}$ & $\begin{array}{l}0.788 \\
(0.00)\end{array}$ & $\begin{array}{l}-17.705 \\
(0.00)\end{array}$ & $\begin{array}{l}0.160 \\
(0.00)\end{array}$ & $\begin{array}{l}0.165 \\
(0.00)\end{array}$ & $\begin{array}{l}-0.964 \\
(0.00)\end{array}$ & $\begin{array}{l}0.507 \\
(0.00)\end{array}$ \\
\hline Peugeot & - & - & - & - & - & - & - \\
\hline Pinault-PR & $\begin{array}{l}-0.998 \\
(0.00)\end{array}$ & $\begin{array}{l}0.995 \\
(0.00)\end{array}$ & $\begin{array}{l}-18.535 \\
(0.00)\end{array}$ & $\begin{array}{l}-0.138 \\
(0.02)\end{array}$ & $\begin{array}{l}0.056 \\
(0.31)\end{array}$ & $\begin{array}{l}-0.983 \\
(0.00)\end{array}$ & $\begin{array}{l}0.093 \\
(0.40)\end{array}$ \\
\hline Renault & $\begin{array}{l}-0.357 \\
(0.54)\end{array}$ & $\begin{array}{l}0.448 \\
(0.45)\end{array}$ & $\begin{array}{l}-3.117 \\
(0.37)\end{array}$ & $\begin{array}{l}0.188 \\
(0.48)\end{array}$ & $\begin{array}{l}0.455 \\
(0.17)\end{array}$ & $\begin{array}{l}0.677 \\
(0.07)\end{array}$ & $\begin{array}{l}-0.005 \\
(0.95)\end{array}$ \\
\hline Saint-Gobain & $\begin{array}{l}0.857 \\
(0.00)\end{array}$ & $\begin{array}{l}-0.800 \\
(0.00)\end{array}$ & $\begin{array}{l}-1.246 \\
(0.04)\end{array}$ & $\begin{array}{l}0.244 \\
(0.01)\end{array}$ & $\begin{array}{l}0.101 \\
(0.19)\end{array}$ & $\begin{array}{l}0.872 \\
(0.00)\end{array}$ & $\begin{array}{l}0.318 \\
(0.35)\end{array}$ \\
\hline Sanofi Synthelabo & $\begin{array}{l}-0.738 \\
(0.56)\end{array}$ & $\begin{array}{l}0.739 \\
(0.56)\end{array}$ & $\begin{array}{l}-4.201 \\
(0.14)\end{array}$ & $\begin{array}{l}0.033 \\
(0.73)\end{array}$ & $\begin{array}{l}0.377 \\
(0.02)\end{array}$ & $\begin{array}{l}0.582 \\
(0.05)\end{array}$ & $\begin{array}{l}0.49 \\
(0.05)\end{array}$ \\
\hline Schneider & $\begin{array}{l}0.400 \\
(0.16)\end{array}$ & $\begin{array}{l}-0.172 \\
(0.55)\end{array}$ & $\begin{array}{l}-4.342 \\
(0.00)\end{array}$ & $\begin{array}{l}-0.349 \\
(0.04)\end{array}$ & $\begin{array}{l}0.430 \\
(0.02)\end{array}$ & $\begin{array}{l}0.553 \\
(0.00)\end{array}$ & $\begin{array}{l}1.218 \\
(0.00)\end{array}$ \\
\hline Société Générale & $\begin{array}{l}-0.618 \\
(0.00) \\
\end{array}$ & $\begin{array}{l}0.713 \\
(0.00) \\
\end{array}$ & $\begin{array}{l}-6.814 \\
(0.01) \\
\end{array}$ & $\begin{array}{l}0.011 \\
(0.92) \\
\end{array}$ & $\begin{array}{l}0.010 \\
(0.97) \\
\end{array}$ & $\begin{array}{l}0.245 \\
(0.43) \\
\end{array}$ & $\begin{array}{l}-1.230 \\
(0.00) \\
\end{array}$ \\
\hline Sodexho & - & - & - & - & - & - & - \\
\hline ST-Microelectronics & $\begin{array}{l}-0.543 \\
(0.01)\end{array}$ & $\begin{array}{l}0.571 \\
(0.01)\end{array}$ & $\begin{array}{l}-0.650 \\
(0.01)\end{array}$ & $\begin{array}{l}-0.097 \\
(0.20)\end{array}$ & $\begin{array}{l}0.092 \\
(0.26)\end{array}$ & $\begin{array}{l}0.922 \\
(0.00)\end{array}$ & $\begin{array}{l}-0.738 \\
(0.02)\end{array}$ \\
\hline Suez Lyon.Des Eaux & $\begin{array}{l}-0.220 \\
(0.95)\end{array}$ & $\begin{array}{l}0.218 \\
(0.95)\end{array}$ & $\begin{array}{l}-4.613 \\
(0.01)\end{array}$ & $\begin{array}{l}0.180 \\
(0.91)\end{array}$ & $\begin{array}{l}-0.432 \\
(0.45)\end{array}$ & $\begin{array}{l}0.011 \\
(0.97)\end{array}$ & $\begin{array}{l}0.051 \\
(0.99)\end{array}$ \\
\hline Tf1 & $\begin{array}{l}-0.598 \\
(0.00)\end{array}$ & $\begin{array}{l}0.676 \\
(0.00)\end{array}$ & $\begin{array}{l}-0.717 \\
(0.11)\end{array}$ & $\begin{array}{l}-0.202 \\
(0.09)\end{array}$ & $\begin{array}{l}0.032 \\
(0.62)\end{array}$ & $\begin{array}{l}0.922 \\
(0.00)\end{array}$ & $\begin{array}{l}0.455 \\
(0.00)\end{array}$ \\
\hline Thales & $\begin{array}{l}-0.462 \\
(0.42)\end{array}$ & $\begin{array}{l}0.461 \\
(0.42)\end{array}$ & $\begin{array}{l}-7.617 \\
(0.03)\end{array}$ & $\begin{array}{l}0.115 \\
(0.21)\end{array}$ & $\begin{array}{l}-0.088 \\
(0.51)\end{array}$ & $\begin{array}{l}0.154 \\
(0.70)\end{array}$ & $\begin{array}{l}0.674 \\
(0.00)\end{array}$ \\
\hline Thomson & $\begin{array}{l}-0.488 \\
(0.11)\end{array}$ & $\begin{array}{l}0.500 \\
(0.10)\end{array}$ & $\begin{array}{l}-4.384 \\
(0.00)\end{array}$ & $\begin{array}{l}0.089 \\
(0.47)\end{array}$ & $\begin{array}{l}0.403 \\
(0.04)\end{array}$ & $\begin{array}{l}0.462 \\
(0.00)\end{array}$ & $\begin{array}{l}-5.444 \\
(0.00)\end{array}$ \\
\hline Total Fina Elf & $\begin{array}{l}0.159 \\
(0.62)\end{array}$ & $\begin{array}{l}-0.222 \\
(0.48)\end{array}$ & $\begin{array}{l}-0.372 \\
(0.02)\end{array}$ & $\begin{array}{l}-0.070 \\
(0.03)\end{array}$ & $\begin{array}{l}-0.065 \\
(0.08)\end{array}$ & $\begin{array}{l}0.960 \\
(0.00)\end{array}$ & $\begin{array}{l}0.277 \\
(0.00)\end{array}$ \\
\hline Valeo & $\begin{array}{l}0.945 \\
(0.00)\end{array}$ & $\begin{array}{l}-0.820 \\
(0.00)\end{array}$ & $\begin{array}{l}-7.560 \\
(0.00)\end{array}$ & $\begin{array}{l}0.413 \\
(0.00)\end{array}$ & $\begin{array}{l}0.712 \\
(0.00)\end{array}$ & $\begin{array}{l}0.203 \\
(0.17)\end{array}$ & $\begin{array}{l}0.614 \\
(0.00)\end{array}$ \\
\hline Vivendi Environment & $\begin{array}{l}0.752 \\
(0.00)\end{array}$ & $\begin{array}{l}-0.602 \\
(0.00)\end{array}$ & $\begin{array}{l}-1.244 \\
(0.31)\end{array}$ & $\begin{array}{l}0.283 \\
(0.00)\end{array}$ & $\begin{array}{l}0.222 \\
(0.17)\end{array}$ & $\begin{array}{l}0.881 \\
(0.00)\end{array}$ & $\begin{array}{l}0.071 \\
(0.65)\end{array}$ \\
\hline Vivendi Universal & $\begin{array}{l}-0.504 \\
(0.11) \\
\end{array}$ & $\begin{array}{l}0.518 \\
(0.10) \\
\end{array}$ & $\begin{array}{l}-0.554 \\
(0.13) \\
\end{array}$ & $\begin{array}{l}-0.013 \\
(0.84) \\
\end{array}$ & $\begin{array}{l}0.298 \\
(0.13) \\
\end{array}$ & $\begin{array}{l}0.962 \\
(0.00) \\
\end{array}$ & $\begin{array}{l}-0.047 \\
(0.21) \\
\end{array}$ \\
\hline CAC40 & $\begin{array}{l}-0.472 \\
(0.29)\end{array}$ & $\begin{array}{l}0.532 \\
(0.21)\end{array}$ & $\begin{array}{l}-9.261 \\
(0.00)\end{array}$ & $\begin{array}{l}0.156 \\
(0.28)\end{array}$ & $\begin{array}{l}0.311 \\
(0.02)\end{array}$ & $\begin{array}{l}0.053 \\
(0.87)\end{array}$ & $\begin{array}{l}-0.419 \\
(0.55)\end{array}$ \\
\hline \multicolumn{8}{|c|}{$\bar{r}_{t}=\bar{\phi}_{0} \bar{r}_{t-1}+\bar{\phi}_{1} \bar{\varepsilon}_{t-1}+\bar{\varepsilon}_{t}$} \\
\hline
\end{tabular}

Table 2 - This table presents the results of the estimation of model (4) which tests the relation between volatility and overnight announcements. Volatility is estimated with the EGARCH model proposed by Nelson (1991). $\bar{r}_{t}$ and $\bar{h}_{t}$ are respectively the overnight return and conditional volatility. $\bar{N}_{t}$ is the number of overnight news items published by Reuters. P-values (in parentheses) are calculated using Bollerslev and Wooldrige's (1992) robust standard errors. 
Table 3: the intraday relation between volume and news releases during the continuous market

\begin{tabular}{|c|c|c|c|c|c|c|}
\hline Titre & $C$ & $\lambda$ & $\theta_{1}$ & $\theta_{2}$ & Fisher & $R^{2}$ \\
\hline$\overline{\text { Accor }}$ & $\begin{array}{l}9.477 \\
(0.00)\end{array}$ & $\begin{array}{l}0.391 \\
(0.00)\end{array}$ & $\begin{array}{l}-0.887 \\
(0.00)\end{array}$ & $\begin{array}{l}1.037 \\
(0.00)\end{array}$ & $\begin{array}{l}160.550 \\
(0.00)\end{array}$ & 0.053 \\
\hline AGF & $\begin{array}{l}8.414 \\
(0.00)\end{array}$ & $\begin{array}{l}0.359 \\
(0.17)\end{array}$ & $\begin{array}{l}-0.937 \\
(0.00)\end{array}$ & $\begin{array}{l}1.403 \\
(0.00)\end{array}$ & $\begin{array}{l}192.930 \\
(0.00)\end{array}$ & 0.063 \\
\hline Air liquide & $\begin{array}{l}8.375 \\
(0.00)\end{array}$ & $\begin{array}{l}0.679 \\
(0.00)\end{array}$ & $\begin{array}{l}-0.510 \\
(0.00)\end{array}$ & $\begin{array}{l}1.095 \\
(0.00)\end{array}$ & $\begin{array}{l}167.380 \\
(0.00)\end{array}$ & 0.055 \\
\hline Alcatel & $\begin{array}{l}12.182 \\
(0.00)\end{array}$ & $\begin{array}{l}0.327 \\
(0.00)\end{array}$ & $\begin{array}{l}0.394 \\
(0.00)\end{array}$ & $\begin{array}{l}0.620 \\
(0.00)\end{array}$ & $\begin{array}{l}79.440 \\
(0.00)\end{array}$ & 0.027 \\
\hline Alstom & $\begin{array}{l}10.031 \\
(0.00)\end{array}$ & $\begin{array}{l}0.880 \\
(0.00)\end{array}$ & $\begin{array}{l}-0.550 \\
(0.00)\end{array}$ & $\begin{array}{l}0.881 \\
(0.00)\end{array}$ & $\begin{array}{l}81.460 \\
(0.00)\end{array}$ & 0.027 \\
\hline Aventis & $\begin{array}{l}10.662 \\
(0.00) \\
\end{array}$ & $\begin{array}{l}0.091 \\
(0.34) \\
\end{array}$ & $\begin{array}{l}-0.320 \\
(0.00) \\
\end{array}$ & $\begin{array}{l}0.774 \\
(0.00) \\
\end{array}$ & $\begin{array}{l}116.310 \\
(0.00)\end{array}$ & 0.039 \\
\hline Axa & $\begin{array}{l}10.967 \\
(0.00)\end{array}$ & $\begin{array}{l}0.659 \\
(0.00)\end{array}$ & $\begin{array}{l}-0.316 \\
(0.00)\end{array}$ & $\begin{array}{l}0.838 \\
(0.00)\end{array}$ & $\begin{array}{l}73.510 \\
(0.00)\end{array}$ & 0.025 \\
\hline Bnp Paribas & $\begin{array}{l}10.293 \\
(0.00)\end{array}$ & $\begin{array}{l}0.473 \\
(0.00)\end{array}$ & $\begin{array}{l}-0.414 \\
(0.00)\end{array}$ & $\begin{array}{l}0.814 \\
(0.00)\end{array}$ & $\begin{array}{l}124.330 \\
(0.00)\end{array}$ & 0.041 \\
\hline Cap Gemini & $\begin{array}{l}9.490 \\
(0.00)\end{array}$ & $\begin{array}{l}0.905 \\
(0.00)\end{array}$ & $\begin{array}{l}0.063 \\
(0.00)\end{array}$ & $\begin{array}{l}0.861 \\
(0.30)\end{array}$ & $\begin{array}{l}89.920 \\
(0.00)\end{array}$ & 0.030 \\
\hline Carrefour & $\begin{array}{l}10.343 \\
(0.00)\end{array}$ & $\begin{array}{l}0.366 \\
(0.00)\end{array}$ & $\begin{array}{l}-0.424 \\
(0.00)\end{array}$ & $\begin{array}{l}0.952 \\
(0.00)\end{array}$ & $\begin{array}{l}153.440 \\
(0.00)\end{array}$ & 0.051 \\
\hline Casino & $\begin{array}{l}8.051 \\
(0.00)\end{array}$ & $\begin{array}{l}0.537 \\
(0.00)\end{array}$ & $\begin{array}{l}-0.718 \\
(0.00)\end{array}$ & $\begin{array}{l}1.165 \\
(0.00)\end{array}$ & $\begin{array}{l}134.900 \\
(0.00)\end{array}$ & 0.045 \\
\hline Crédit Lyonnais & $\begin{array}{l}9.237 \\
(0.00)\end{array}$ & $\begin{array}{l}0.601 \\
(0.00)\end{array}$ & $\begin{array}{l}-0.378 \\
(0.00)\end{array}$ & $\begin{array}{l}1.248 \\
(0.00)\end{array}$ & $\begin{array}{l}125.990 \\
(0.00)\end{array}$ & 0.042 \\
\hline Danone & $\begin{array}{l}9.165 \\
(0.00)\end{array}$ & $\begin{array}{l}0.527 \\
(0.00)\end{array}$ & $\begin{array}{l}-0.678 \\
(0.00)\end{array}$ & $\begin{array}{l}0.966 \\
(0.00)\end{array}$ & $\begin{array}{l}172.810 \\
(0.00)\end{array}$ & 0.057 \\
\hline Dassault & $\begin{array}{l}8.350 \\
(0.00)\end{array}$ & $\begin{array}{l}0.48678 \\
-0.010\end{array}$ & $\begin{array}{l}-0.310 \\
(0.00)\end{array}$ & $\begin{array}{l}1.202 \\
(0.00)\end{array}$ & $\begin{array}{l}110.470 \\
(0.00)\end{array}$ & 0.037 \\
\hline Dexia & $\begin{array}{l}8.801 \\
(0.00)\end{array}$ & $\begin{array}{l}0.916 \\
(0.00)\end{array}$ & $\begin{array}{l}-0.467 \\
(0.00)\end{array}$ & $\begin{array}{l}1.077 \\
(0.00)\end{array}$ & $\begin{array}{l}49.220 \\
(0.00)\end{array}$ & 0.017 \\
\hline Eads & $\begin{array}{l}10.015 \\
(0.00)\end{array}$ & $\begin{array}{l}0.637 \\
(0.00)\end{array}$ & $\begin{array}{l}-0.350 \\
(0.00)\end{array}$ & $\begin{array}{l}1.145 \\
(0.00)\end{array}$ & $\begin{array}{l}122.840 \\
(0.00)\end{array}$ & 0.041 \\
\hline Equant & $\begin{array}{l}9.471 \\
(0.00)\end{array}$ & $\begin{array}{l}0.547 \\
(0.02)\end{array}$ & $\begin{array}{l}-0.320 \\
(0.00)\end{array}$ & $\begin{array}{l}1.181 \\
(0.00)\end{array}$ & $\begin{array}{l}90.810 \\
(0.00)\end{array}$ & 0.031 \\
\hline France Télécom & $\begin{array}{l}11.294 \\
(0.00)\end{array}$ & $\begin{array}{l}0.261 \\
(0.00)\end{array}$ & $\begin{array}{l}0.214 \\
(0.00)\end{array}$ & $\begin{array}{l}0.713 \\
(0.00)\end{array}$ & $\begin{array}{l}69.590 \\
(0.00)\end{array}$ & 0.023 \\
\hline Lafarge & $\begin{array}{l}9.126 \\
(0.00)\end{array}$ & $\begin{array}{l}0.534 \\
(0.00)\end{array}$ & $\begin{array}{l}-0.785 \\
(0.00)\end{array}$ & $\begin{array}{l}0.998 \\
(0.00)\end{array}$ & $\begin{array}{l}154.830 \\
(0.00)\end{array}$ & 0.051 \\
\hline Lagardère & $\begin{array}{l}8.856 \\
(0.00)\end{array}$ & $\begin{array}{l}0.563 \\
(0.00)\end{array}$ & $\begin{array}{l}-0.495 \\
(0.00)\end{array}$ & $\begin{array}{l}1.464 \\
(0.00)\end{array}$ & $\begin{array}{l}184.730 \\
(0.00)\end{array}$ & 0.060 \\
\hline L'oréal & $\begin{array}{l}9.782 \\
(0.00)\end{array}$ & $\begin{array}{l}0.299 \\
(0.08)\end{array}$ & $\begin{array}{l}-0.381 \\
(0.00)\end{array}$ & $\begin{array}{l}0.878 \\
(0.00)\end{array}$ & $\begin{array}{l}134.080 \\
(0.00)\end{array}$ & 0.044 \\
\hline LVMH & $\begin{array}{l}10.012 \\
(0.00)\end{array}$ & $\begin{array}{l}0.358 \\
(0.00)\end{array}$ & $\begin{array}{l}-0.515 \\
(0.00)\end{array}$ & $\begin{array}{l}0.993 \\
(0.00)\end{array}$ & $\begin{array}{l}148.780 \\
(0.00)\end{array}$ & 0.049 \\
\hline Michelin & $\begin{array}{l}8.606 \\
(0.00)\end{array}$ & $\begin{array}{l}0.821 \\
(0.00)\end{array}$ & $\begin{array}{l}-0.671 \\
(0.00)\end{array}$ & $\begin{array}{l}1.364 \\
(0.00)\end{array}$ & $\begin{array}{l}142.74 \\
(0.00)\end{array}$ & 0.047 \\
\hline
\end{tabular}


Table 3 (continued)

\begin{tabular}{|c|c|c|c|c|c|c|}
\hline titre & $C$ & $\lambda$ & $\theta_{1}$ & $\theta_{2}$ & Fisher & $R^{2}$ \\
\hline Orange & $\begin{array}{l}11.748 \\
(0.00)\end{array}$ & $\begin{array}{l}0.584 \\
(0.00)\end{array}$ & $\begin{array}{l}-0.266 \\
(0.00)\end{array}$ & $\begin{array}{l}0.754 \\
(0.00)\end{array}$ & $\begin{array}{l}46.890 \\
(0.00)\end{array}$ & 0.018 \\
\hline Peugeot & $\begin{array}{l}8.943 \\
(0.00)\end{array}$ & $\begin{array}{l}0.501 \\
(0.01)\end{array}$ & $\begin{array}{l}-0.829 \\
(0.00)\end{array}$ & $\begin{array}{l}1.013 \\
(0.00)\end{array}$ & $\begin{array}{l}91.580 \\
(0.00)\end{array}$ & 0.031 \\
\hline Prinault-PR & $\begin{array}{l}8.539 \\
(0.00)\end{array}$ & $\begin{array}{l}0.718 \\
(0.00)\end{array}$ & $\begin{array}{l}-0.618 \\
(0.00)\end{array}$ & $\begin{array}{l}1.191 \\
(0.00)\end{array}$ & $\begin{array}{l}163.080 \\
(0.00)\end{array}$ & 0.054 \\
\hline Renault & $\begin{array}{l}9.500 \\
(0.00)\end{array}$ & $\begin{array}{l}0.339 \\
(0.00)\end{array}$ & $\begin{array}{l}-0.434 \\
(0.00)\end{array}$ & $\begin{array}{l}1.117 \\
(0.00)\end{array}$ & $\begin{array}{l}140.270 \\
(0.00)\end{array}$ & 0.046 \\
\hline Saint-Gobin & $\begin{array}{l}8.403 \\
(0.00)\end{array}$ & $\begin{array}{l}0.580 \\
(0.00)\end{array}$ & $\begin{array}{l}-0.704 \\
(0.00)\end{array}$ & $\begin{array}{l}1.076 \\
(0.00)\end{array}$ & $\begin{array}{l}149.620 \\
(0.00)\end{array}$ & 0.049 \\
\hline Sanofi Synthelabo & $\begin{array}{l}10.347 \\
(0.00)\end{array}$ & $\begin{array}{l}0.340 \\
(0.00)\end{array}$ & $\begin{array}{l}-0.347 \\
(0.00)\end{array}$ & $\begin{array}{l}0.852 \\
(0.00)\end{array}$ & $\begin{array}{l}117.310 \\
(0.00)\end{array}$ & 0.039 \\
\hline Schneider & $\begin{array}{l}9.536 \\
(0.00)\end{array}$ & $\begin{array}{l}0.880 \\
(0.00)\end{array}$ & $\begin{array}{l}-0.925 \\
(0.00)\end{array}$ & $\begin{array}{c}0.994 \\
(0.00)\end{array}$ & $\begin{array}{l}167.880 \\
(0.00)\end{array}$ & 0.056 \\
\hline Société Générale & $\begin{array}{l}10.069 \\
(0.00)\end{array}$ & $\begin{array}{l}0.182 \\
(0.29)\end{array}$ & $\begin{array}{l}-0.411 \\
(0.00)\end{array}$ & $\begin{array}{l}1.048 \\
(0.00)\end{array}$ & $\begin{array}{l}155.040 \\
(0.00)\end{array}$ & 0.051 \\
\hline Sodexho & $\begin{array}{l}8.427 \\
(0.00)\end{array}$ & $\begin{array}{l}0.901 \\
(0.00)\end{array}$ & $\begin{array}{l}-0.943 \\
(0.00)\end{array}$ & $\begin{array}{l}1.357 \\
(0.00)\end{array}$ & $\begin{array}{l}123.650 \\
(0.00)\end{array}$ & 0.041 \\
\hline ST-Microelectronics & $\begin{array}{l}11.573 \\
(0.00)\end{array}$ & $\begin{array}{l}0.475 \\
(0.01)\end{array}$ & $\begin{array}{l}0.030 \\
(0.58)\end{array}$ & $\begin{array}{l}0.571 \\
(0.00)\end{array}$ & $\begin{array}{l}37.960 \\
(0.00)\end{array}$ & 0.013 \\
\hline Suez Lyon.Des Eaux & $\begin{array}{l}10.241 \\
(0.00)\end{array}$ & $\begin{array}{l}0.042 \\
(0.75)\end{array}$ & $\begin{array}{l}-0.661 \\
(0.00)\end{array}$ & $\begin{array}{l}0.905 \\
(0.00)\end{array}$ & $\begin{array}{l}91.320 \\
(0.00)\end{array}$ & 0.031 \\
\hline TF1 & $\begin{array}{l}9.450 \\
(0.00)\end{array}$ & $\begin{array}{l}0.260 \\
(0.00)\end{array}$ & $\begin{array}{l}-0.422 \\
(0.00)\end{array}$ & $\begin{array}{l}1.065 \\
(0.00)\end{array}$ & $\begin{array}{l}119.300 \\
(0.00)\end{array}$ & 0.040 \\
\hline Thales & $\begin{array}{l}8.313 \\
(0.00)\end{array}$ & $\begin{array}{l}0.646 \\
(0.00)\end{array}$ & $\begin{array}{l}-0.729 \\
(0.00)\end{array}$ & $\begin{array}{l}1.295 \\
(0.00)\end{array}$ & $\begin{array}{l}139.100 \\
(0.00)\end{array}$ & 0.046 \\
\hline Thomson & $\begin{array}{l}9.485 \\
(0.00)\end{array}$ & $\begin{array}{l}0.752 \\
(0.02)\end{array}$ & $\begin{array}{l}-0.100 \\
(0.12)\end{array}$ & $\begin{array}{l}1.042 \\
(0.00)\end{array}$ & $\begin{array}{l}88.350 \\
(0.00)\end{array}$ & 0.030 \\
\hline Total Fina Elf & $\begin{array}{l}10.645 \\
(0.00)\end{array}$ & $\begin{array}{l}0.072 \\
(0.40)\end{array}$ & $\begin{array}{l}-0.076 \\
(0.10)\end{array}$ & $\begin{array}{l}1.031 \\
(0.00)\end{array}$ & $\begin{array}{l}165.120 \\
(0.00)\end{array}$ & 0.054 \\
\hline Valeo & $\begin{array}{l}8.304 \\
(0.00)\end{array}$ & $\begin{array}{l}0.601 \\
(0.00)\end{array}$ & $\begin{array}{l}-1.004 \\
(0.00)\end{array}$ & $\begin{array}{l}1.425 \\
(0.00)\end{array}$ & $\begin{array}{l}158.280 \\
(0.00)\end{array}$ & 0.053 \\
\hline Vivendi Environment & $\begin{array}{l}8.810 \\
(0.00)\end{array}$ & $\begin{array}{l}0.791 \\
(0.00)\end{array}$ & $\begin{array}{l}-1.030 \\
(0.00)\end{array}$ & $\begin{array}{l}1.321 \\
(0.00)\end{array}$ & $\begin{array}{l}120.140 \\
(0.00)\end{array}$ & 0.040 \\
\hline Vivendi Universal & $\begin{array}{l}11.140 \\
(0.00)\end{array}$ & $\begin{array}{l}0.237 \\
(0.00)\end{array}$ & $\begin{array}{l}-0.111 \\
(0.02)\end{array}$ & $\begin{array}{l}1.096 \\
(0.00)\end{array}$ & $\begin{array}{l}166.450 \\
(0.00)\end{array}$ & 0.055 \\
\hline CAC40 & $\begin{array}{l}10.853 \\
(0.00)\end{array}$ & $\begin{array}{l}0.060 \\
(0.00)\end{array}$ & $\begin{array}{l}0.021 \\
(0.55)\end{array}$ & $\begin{array}{l}0.811 \\
(0.00)\end{array}$ & $\begin{array}{l}188.02 \\
(0.00)\end{array}$ & 0.062 \\
\hline
\end{tabular}

Table 3 - This table presents the results of the estimation of model (5) which tests the intraday relation between trading volume and news releases during the continuous market. $\mathrm{V}_{\mathrm{t}}$ is the trading volume the 15-minute period t. $\mathrm{N}_{\mathrm{t}}$ is the number of news items published by Reuters during each 15 -interval t. DO and DC are two dummy variables accounting respectively for the first 15-minute period of the trading day and for the last 15-minute period. 
Table 4: the relation between volume and overnight announcements

\begin{tabular}{|c|c|c|c|c|}
\hline Titre & $C$ & $\lambda$ & Fisher & $R^{2}$ \\
\hline Accor & $\begin{array}{l}9.036 \\
(0.00)\end{array}$ & $\begin{array}{l}0.524 \\
(0.00)\end{array}$ & $\begin{array}{l}8.620 \\
(0.00)\end{array}$ & 0.033 \\
\hline AGF & $\begin{array}{l}8.159 \\
(0.00)\end{array}$ & $\begin{array}{l}0.088 \\
(0.71)\end{array}$ & $\begin{array}{l}0.140 \\
(0.71)\end{array}$ & 0.001 \\
\hline Air liquide & $\begin{array}{l}8.169 \\
(0.00)\end{array}$ & $\begin{array}{l}0.140 \\
(0.13)\end{array}$ & $\begin{array}{l}0.800 \\
(0.13)\end{array}$ & 0.003 \\
\hline Alcatel & $\begin{array}{l}11.858 \\
(0.00)\end{array}$ & $\begin{array}{l}0.141 \\
(0.00)\end{array}$ & $\begin{array}{l}11.250 \\
(0.00)\end{array}$ & 0.043 \\
\hline Alstom & $\begin{array}{l}9.588 \\
(0.00)\end{array}$ & $\begin{array}{l}0.778 \\
(0.00)\end{array}$ & $\begin{array}{l}26.270 \\
(0.00)\end{array}$ & 0.095 \\
\hline Aventis & $\begin{array}{l}10.093 \\
(0.00)\end{array}$ & $\begin{array}{l}0.078 \\
(0.36)\end{array}$ & $\begin{array}{l}0.830 \\
(0.36)\end{array}$ & 0.003 \\
\hline Axa & $\begin{array}{l}10.640 \\
(0.00)\end{array}$ & $\begin{array}{l}0.041 \\
(0.81)\end{array}$ & $\begin{array}{l}0.050 \\
(0.81)\end{array}$ & $(0.00)$ \\
\hline Bnp Paribas & $\begin{array}{l}9.663 \\
(0.00)\end{array}$ & $\begin{array}{l}0.255 \\
(0.05)\end{array}$ & $\begin{array}{l}3.750 \\
(0.05)\end{array}$ & 0.015 \\
\hline Cap Gemini & $\begin{array}{l}9.138 \\
(0.00)\end{array}$ & $\begin{array}{l}0.513 \\
(0.00)\end{array}$ & $\begin{array}{l}15.900 \\
(0.00)\end{array}$ & 0.060 \\
\hline Carrefour & $\begin{array}{l}9.978 \\
(0.00)\end{array}$ & $\begin{array}{l}0.302 \\
(0.00)\end{array}$ & $\begin{array}{l}6.820 \\
(0.00)\end{array}$ & 0.027 \\
\hline Casino & $\begin{array}{l}7.667 \\
(0.00)\end{array}$ & $\begin{array}{l}0.614 \\
(0.00)\end{array}$ & $\begin{array}{l}8.620 \\
(0.00)\end{array}$ & 0.033 \\
\hline Crédit Lyonnais & $\begin{array}{l}9.516 \\
(0.00)\end{array}$ & $\begin{array}{l}0.358 \\
(0.00)\end{array}$ & $\begin{array}{l}7.430 \\
(0.00)\end{array}$ & 0.029 \\
\hline Danone & $\begin{array}{l}8.576 \\
(0.00)\end{array}$ & $\begin{array}{l}0.296 \\
(0.00)\end{array}$ & $\begin{array}{l}9.970 \\
(0.00)\end{array}$ & 0.038 \\
\hline Dassault & $\begin{array}{l}8.424 \\
(0.00)\end{array}$ & $\begin{array}{l}0.578 \\
(0.00)\end{array}$ & $\begin{array}{l}7.650 \\
(0.00)\end{array}$ & 0.030 \\
\hline Dexia & $\begin{array}{l}8.774 \\
(0.00)\end{array}$ & $\begin{array}{l}-0.379 \\
(0.22)\end{array}$ & $\begin{array}{l}1.460 \\
(0.22)\end{array}$ & 0.006 \\
\hline Eads & $\begin{array}{l}9.991 \\
(0.00)\end{array}$ & $\begin{array}{l}0.174 \\
(0.23)\end{array}$ & $\begin{array}{l}1.440 \\
(0.23)\end{array}$ & 0.006 \\
\hline Equant & $\begin{array}{l}9.329 \\
(0.00)\end{array}$ & $\begin{array}{l}0.326 \\
(0.14)\end{array}$ & $\begin{array}{l}2.160 \\
(0.14)\end{array}$ & 0.002 \\
\hline France Télécom & $\begin{array}{l}10.914 \\
(0.00)\end{array}$ & $\begin{array}{l}0.151 \\
(0.02)\end{array}$ & $\begin{array}{l}5.240 \\
(0.02)\end{array}$ & 0.021 \\
\hline Lafarge & $\begin{array}{l}8.512 \\
(0.00)\end{array}$ & $\begin{array}{l}0.435 \\
(0.00)\end{array}$ & $\begin{array}{l}16.470 \\
(0.00)\end{array}$ & 0.002 \\
\hline Lagardère & $\begin{array}{l}8.939 \\
(0.00)\end{array}$ & $\begin{array}{l}0.141 \\
(0.53)\end{array}$ & $\begin{array}{l}0.380 \\
(0.53)\end{array}$ & 0.002 \\
\hline Loréal & $\begin{array}{l}9.532 \\
(0.00)\end{array}$ & $\begin{array}{l}0.874 \\
(0.00)\end{array}$ & $\begin{array}{l}14.760 \\
(0.00)\end{array}$ & 0.056 \\
\hline LVMH & $\begin{array}{l}9.553 \\
(0.00)\end{array}$ & $\begin{array}{l}0.230 \\
(0.05)\end{array}$ & $\begin{array}{l}3.580 \\
(0.05)\end{array}$ & 0.014 \\
\hline Michelin & $\begin{array}{l}8.677 \\
(0.00)\end{array}$ & $\begin{array}{l}0.459 \\
(0.01)\end{array}$ & $\begin{array}{l}6.130 \\
(0.01)\end{array}$ & 0.024 \\
\hline
\end{tabular}




\begin{tabular}{|c|c|c|c|c|}
\hline Titre & $C$ & $\lambda$ & Fisher & $R^{2}$ \\
\hline Orange & $\begin{array}{l}11.068 \\
(0.00)\end{array}$ & $\begin{array}{l}0.443 \\
(0.01)\end{array}$ & $\begin{array}{l}6.420 \\
(0.01)\end{array}$ & 0.028 \\
\hline Peugeot & $\begin{array}{l}8.323 \\
(0.00)\end{array}$ & $\begin{array}{l}0.291 \\
(0.34)\end{array}$ & $\begin{array}{l}0.890 \\
(0.34)\end{array}$ & 0.004 \\
\hline Prinault-PR & $\begin{array}{l}8.070 \\
(0.00)\end{array}$ & $\begin{array}{l}0.225 \\
(0.09)\end{array}$ & $\begin{array}{l}2.830 \\
(0.09)\end{array}$ & 0.011 \\
\hline Renault & $\begin{array}{l}9.151 \\
(0.00)\end{array}$ & $\begin{array}{l}0.100 \\
(0.17)\end{array}$ & $\begin{array}{l}1.820 \\
(0.17)\end{array}$ & 0.007 \\
\hline Saint-Gobin & $\begin{array}{l}8.081 \\
(0.00)\end{array}$ & $\begin{array}{l}0.464 \\
(0.00)\end{array}$ & $\begin{array}{l}10.710 \\
(0.00)\end{array}$ & 0.041 \\
\hline Sanofi Synthelabo & $\begin{array}{l}9.781 \\
(0.00)\end{array}$ & $\begin{array}{l}0.536 \\
(0.00)\end{array}$ & $\begin{array}{l}9.360 \\
(0.00)\end{array}$ & 0.036 \\
\hline Schneider & $\begin{array}{l}8.740 \\
(0.00)\end{array}$ & $\begin{array}{l}0.539 \\
(0.00)\end{array}$ & $\begin{array}{l}24.820 \\
(0.00)\end{array}$ & 0.091 \\
\hline Société Générale & $\begin{array}{l}9.418 \\
(0.00)\end{array}$ & $\begin{array}{l}0.051 \\
(0.76)\end{array}$ & $\begin{array}{l}0.090 \\
(0.76)\end{array}$ & $(0.00)$ \\
\hline Sodexho & $\begin{array}{l}8.015 \\
(0.00)\end{array}$ & $\begin{array}{l}0.476 \\
(0.04)\end{array}$ & $\begin{array}{l}4.120 \\
(0.04)\end{array}$ & 0.016 \\
\hline ST-Microelectronics & $\begin{array}{l}10.800 \\
(0.00)\end{array}$ & $\begin{array}{l}1.333 \\
(0.00)\end{array}$ & $\begin{array}{l}17.950 \\
(0.00)\end{array}$ & 0.067 \\
\hline Suez Lyon.Des Eaux & $\begin{array}{l}9.915 \\
(0.00)\end{array}$ & $\begin{array}{l}-0.175 \\
(0.30)\end{array}$ & $\begin{array}{l}1.050 \\
(0.30)\end{array}$ & 0.004 \\
\hline TF1 & $\begin{array}{l}9.116 \\
(0.00)\end{array}$ & $\begin{array}{l}0.335 \\
(0.01)\end{array}$ & $\begin{array}{l}5.880 \\
(0.01)\end{array}$ & 0.023 \\
\hline Thales & $\begin{array}{l}8.333 \\
(0.00)\end{array}$ & $\begin{array}{l}0.487 \\
(0.00)\end{array}$ & $\begin{array}{l}6.920 \\
(0.00)\end{array}$ & 0.027 \\
\hline Thomson & $\begin{array}{l}9.535 \\
(0.00)\end{array}$ & $\begin{array}{l}0.102 \\
(0.85)\end{array}$ & $\begin{array}{l}0.030 \\
(0.85)\end{array}$ & $(0.00)$ \\
\hline Total Fina Elf & $\begin{array}{l}10.117 \\
(0.00)\end{array}$ & $\begin{array}{l}0.071 \\
(0.60)\end{array}$ & $\begin{array}{l}0.270 \\
(0.60)\end{array}$ & 0.001 \\
\hline Valeo & $\begin{array}{l}7.702 \\
(0.00)\end{array}$ & $\begin{array}{l}0.566 \\
(0.00)\end{array}$ & $\begin{array}{l}15.710 \\
(0.00)\end{array}$ & 0.059 \\
\hline Vivendi Environment & $\begin{array}{l}8.364 \\
(0.00)\end{array}$ & $\begin{array}{l}0.457 \\
(0.00)\end{array}$ & $\begin{array}{l}13.460 \\
(0.00)\end{array}$ & 0.051 \\
\hline Vivendi Universal & $\begin{array}{l}10.781 \\
(0.00)\end{array}$ & $\begin{array}{l}0.085 \\
(0.16)\end{array}$ & $\begin{array}{l}1.920 \\
(0.16)\end{array}$ & 0.008 \\
\hline CAC40 & $\begin{array}{l}10.319 \\
(0.00)\end{array}$ & $\begin{array}{l}0.039 \\
(0.00)\end{array}$ & $\begin{array}{l}9.71 \\
(0.00)\end{array}$ & 0.037 \\
\hline
\end{tabular}

Table 4 - This table presents the results of the estimation of model (6) which tests the relation between trading volume and overnight announcements. $\bar{V}_{t}$ is the opening trading volume at the day t. $\bar{N}_{t}$ is the number of overnight news items published by Reuters. 


\subsection{Public information arrival and trading volume}

Table 3 summarizes the results of the estimation of model (5). We observe that the coefficients of the dummy variables are generally significant which confirm the intraday U-shaped pattern of volume. Moreover, the results show that the coefficient of our informational proxy is significantly positive for 36 firms out of 41, which represents $88 \%$. This finding highlights a strong relationship between the rate of public information flow and trading volume, which confirms the MDH hypothesis.

The results of the estimation of model (6) are given in Table 4. The linear regression reveals that the coefficient of the informational variable $N_{t}$ is positive and significant for 26 out of the 41 firms of our sample, which represents $64 \%$. The results for the CAC40 index confirm this strong positive relationship between trading volume and the information flow. This finding show that the number of news items published during the closing period impacts significantly the trading volume at the opening.

In conclusion, the relationship between the information flow and trading volume seems stronger than the relationship between the information flow and stock price volatility. This result can be explained by the fact that the trading volume may be abnormally high even around "no news" announcements which do not cause a significant price change. Indeed, several empirical studies highlight an abnormal trading volume even in the absence of price changes (Beaver (1968), Bamber and Cheon (1995), Kandel and Pearson (1995), etc.). Theoretically, Blume et al. (1994) explain this empirical result by the fact that volume has a limit distribution that is nondegenerate. The difference in investors' expectations, the difference in the degree of risk aversion among investors, the differential interpretation of information and the publicity effect can explain this phenomenon.

\section{CONCLUSION}

The goal of this paper is to examine the relationship between the information flow and market activity. The information flow is measured as the number of news items recorded by Reuters. Our results reveal a strong relation between trading volume and public information flow and a moderate relation between the information flow and stock returns volatility. These results are available for the CAC40 Index as well as for individual stocks. Moreover, our findings show that high opening market activity is explained by both microstructure factors (Euronext opening protocol) and informational causes i.e. accumulation of public information during the closing period.

Our study confirms partially the mixture distribution hypothesis. However, the moderate relationship between the information flow and stock price volatility can be attributed to the choice of our informational proxy which focuses only on public information. Further research should develop more sophisticated information measure in order to take into account private information.

\section{AUTHOR INFORMATION}

Dr Alexis Cellier holds a Ph.D in Finance (Management) from Fucam (Catholic university of Mons Belgium) and University of Perpignan (France). His research is in the fields of market microstructure and information incorporation. He is currently an Associate Professor at the UPEC (Université Paris Est, Créteil).

Dr. Waël Louhichi is currently an Associate Professor of Finance at Rennes 1 University. He was previously an Assistant Professor at Amiens School of Management. He obtained a Ph.D from both Perpignan University (France) and FUCaM (Belgium). He is a researcher at CREM Rennes (UMR 6211 CNRS). His main area of research is market microstructure and has published several articles in national and international journals (Banque \& Marchés, Review of Accounting and Finance, Management Decision, etc.).

\section{REFERENCES}

1. Andersen, T., Bollerslev, T., 1998. Deutsche mark-dollar volatility: intraday activity patterns, macroeconomic announcements, and longer run dependencies. The Journal of Finance 52(1), 219-265. 
2. Bamber, L. S., Cheon, Y., 1995. Differential price and volume reactions to accounting earnings announcements. The Accounting Review 70(3), 417-441.

3. Beaver, W. H., 1968. The information content of annual earnings announcements. Journal of Accounting Research 6, 67-92.

4. $\quad$ Berry, T. D., Howe, K.M., 1994. Public information arrival. The Journal of Finance 49(4), 1331-1346.

5. Blau, B. M., Van Ness, B. F., Van Ness, R. A., 2009. Intraday stealth trading: which trades move prices during periods of high volume? Journal of Financial Research 32(1), 1-21.

6. Blume, L., Easley, D., O’Hara, M., 1994. Market statistics and technical analysis: the role of volume. The Journal of Finance 49(1), 153-181.

7. Bollerslev, T., 1986. Generalized autoregressive conditional heteroskedasticity. Journal of Econometrics 52(3), 5-59.

8. Bollerslev, T., Wooldridge, J.M., 1992. Quasi-maximum likelihood estimation and inference in dynamic models with time varying covariances. Econometric Reviews 11(2), 143-172.

9. Clark, P. K., 1973. A subordinated stochastic process model with finite variance for speculative prices. Econometrica 41(1), 135-155.

10. Culter, D. M., Poterba, J. M., Summers, L. H., 1989. What moves stock prices? Journal of Portfolio management 15(4), 4-12.

11. Epps, T. W., Epps, M. L., 1976. The stochastic dependence of security price changes and transactions volumes: implications for the mixture of distribution hypothesis. Econometrica 44(2), 305-321.

12. Engle, R. F., 1982. Autoregressive conditional heteroskedasticity with estimates of the variance of United Kingdom inflation. Econometrica 50(4), 987-1008.

13. Fair, R. C., 2002. Events that shook the market. Journal of Business 75(4), 713-731.

14. Fama, E. F., 1991. Efficient capital markets: II. The Journal of Finance 46(5), 1575-1617.

15. Jain, P., Joh, G. H., 1985. The dependence between hourly prices and trading volume. Journal of Financial and Quantative Analysis 23(3), 269-283.

16. Kalev P. S., Liu W. M., Pharm, P. K., Jarnecic, E., 2004. Public information arrival and volatility of intraday stock returns. Journal of Banking and Finance 28(6), 1441-1467.

17. Kandel, L., Pearson, N., 1995. Differential interpretation of public signals and trade in speculative markets. Journal of Political Economy 103(4), 831-872.

18. Lamoureux, C. G., Lastrapes W. D., 1990. Heteroskedasticity in stock return data: volume versus GARCH effects. The Journal of Finance 45(1), 221-229.

19. Li, J., Wu, C., 2006. Daily return volatility, bid-ask spreads, and information flow: analyzing the information content of volume. Journal of Business 79(5), 2697-2739.

20. Louhichi, W., 2008. Market reaction to annual earnings announcements: evidence from Euronext Paris. Review of Accounting and Finance 8(1), 102-115.

21. Maillet, B., Michel T., 1997. Mesure de temps, information et distribution des rendements intrajournaliers. Journal de la Société Statistique de Paris 138(4), 89-119.

22. Melvin, M., Yin, X., 2000. Public information arrival, exchange rate volatility, and quote frequency. Economic Journal 110(465), 644-661.

23. Nelson, D. B., 1991. Conditional heteroskedasticity in asset returns: a new approach. Econometrica 59(2), 347-370.

24. Tauchen, G. E., Pitts M., 1983. The price variability-volume relationship on speculative markets. Econometrica 51(2), 485-506.

25. Wood, R., McInish, T., Ord, J., 1985. An investigation of transaction data for NYSE stocks. Journal of Finance 40 (3), 723-741. 
NOTES 